\title{
Light dark matter, naturalness, and the radiative origin of the electroweak scale
}

\author{
Wolfgang Altmannshofer, ${ }^{a}$ William A. Bardeen, ${ }^{b}$ Martin Bauer, ${ }^{b, c}$ Marcela Carena ${ }^{b, c, d}$ \\ and Joseph D. Lykken ${ }^{b}$ \\ ${ }^{a}$ Perimeter Institute for Theoretical Physics, \\ Waterloo, ON, N2L 2Y5, Canada \\ ${ }^{b}$ Theoretical Physics Department, Fermi National Accelerator Laboratory, \\ Batavia, IL 60510, U.S.A. \\ ${ }^{c}$ Enrico Fermi Institute, University of Chicago, \\ Chicago, IL 6063\%, U.S.A. \\ ${ }^{d}$ Kavli Institute for Cosmological Physics, University of Chicago, \\ Chicago, IL 6063\%, U.S.A. \\ E-mail: waltmannshofer@perimeterinstitute.ca, bardeen@fnal.gov, \\ martinbauer@uchicago.edu, carena@fnal.gov, lykken@fnal.gov
}

ABSTRACT: We study classically scale invariant models in which the Standard Model Higgs mass term is replaced in the Lagrangian by a Higgs portal coupling to a complex scalar field of a dark sector. We focus on models that are weakly coupled with the quartic scalar couplings nearly vanishing at the Planck scale. The dark sector contains fermions and scalars charged under dark $\mathrm{SU}(2) \times \mathrm{U}(1)$ gauge interactions. Radiative breaking of the dark gauge group triggers electroweak symmetry breaking through the Higgs portal coupling. Requiring both a Higgs boson mass of $125.5 \mathrm{GeV}$ and stability of the Higgs potential up to the Planck scale implies that the radiative breaking of the dark gauge group occurs at the $\mathrm{TeV}$ scale. We present a particular model which features a long-range abelian dark force. The dominant dark matter component is neutral dark fermions, with the correct thermal relic abundance, and in reach of future direct detection experiments. The model also has lighter stable dark fermions charged under the dark force, with observable effects on galactic-scale structure. Collider signatures include a dark sector scalar boson with mass $\lesssim 250 \mathrm{GeV}$ that decays through mixing with the Higgs boson, and can be detected at the LHC. The Higgs boson, as well as the new scalar, may have significant invisible decays into dark sector particles.

Keywords: Higgs Physics, Beyond Standard Model

ARXIV EPRINT: 1408.3429 


\section{Contents}

1 Introduction 1

2 Motivation 3

3 UV stability and IR instability from dark sectors 4

3.1 UV stability 4

$\begin{array}{lll}3.2 & \text { IR instability } & 6\end{array}$

3.3 The scale of dark matter 8

4 The model $\quad 9$

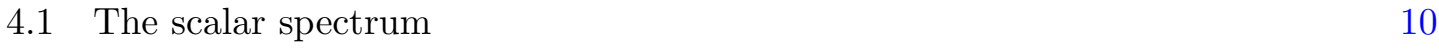

4.2 Vacuum stability in the UV 11

5 Higgs and dark scalar phenomenology 13

6 Dark matter and dark photon phenomenology 17

$\begin{array}{ll}\text { 6.1 Dark matter relic abundance } & 17\end{array}$

$\begin{array}{lll}6.2 & \text { Dark matter direct detection } & 19\end{array}$

6.3 Number of relativistic degrees of freedom in the early universe 20

7 Numerical analysis, discussion, and outlook 21

8 Conclusion $\quad 24$

$\begin{array}{lr}\text { A Effective potential } & 26\end{array}$

$\begin{array}{lr}\text { B Beta functions } & 27\end{array}$

$\begin{array}{ll}\text { C Loop function } & 27\end{array}$

$\begin{array}{ll}\text { D Dark matter annihilation } & 28\end{array}$

\section{Introduction}

The Standard Model (SM) is a renormalizable quantum field theory that makes unambiguous predictions for elementary particle processes over a very large range of energy scales. Apart from a possible metastable vacuum, the SM has no theoretical inconsistencies at least up to the Planck scale at which we expect gravity to become strong and quantum field theories to break down. If this scenario is realized in nature, the Higgs mass parameter seems artificially small compared to the Planck scale. However, in the SM itself 
the Higgs mass parameter is the only explicit scale in the theory, and therefore it is only multiplicatively renormalized [1].

An interesting modification of the SM is given by requiring that the Higgs mass term vanishes at some very high energy (UV) scale; in this case it will not be generated by SM radiative corrections at lower scales either. The tree-level potential has only a quartic term, and the full Lagrangian is classically scale invariant. Electroweak symmetry breaking could be triggered, in principle, by the one-loop corrections to the effective potential

$$
V_{\text {eff }}(h)=\frac{\lambda}{2} h^{4}+B h^{4} \log \left(h^{2} / \mu^{2}\right),
$$

in which $\mu$ denotes the renormalization scale and $B$ is a loop suppressed function of the couplings. Such a possibility has been envisioned by Coleman and Weinberg [2]. A very attractive feature of the Coleman-Weinberg (CW) symmetry breaking mechanism is, that for couplings of order 1 at some renormalization scale in the UV, $\mu=\mu_{\mathrm{UV}}$, the minimum of the potential appears at an exponentially smaller scale

$$
\langle h\rangle \propto \mu_{\mathrm{UV}} e^{-\lambda\left(\mu_{\mathrm{UV}}\right) / B} .
$$

Therefore, similar to the large disparity between the Planck scale and the confinement scale of QCD, the large disparity between the Planck scale and the electroweak scale is explained through renormalization group running [3].

However, in the SM the CW mechanism is ruled out. The dominant contribution to the effective potential comes from the top quark, which renders it unbounded from below, since it enters the coefficient $B$ in (1.1) with a negative sign. In order to overcome the top quark contribution and to reproduce the measured Higgs mass, one would need to extend the SM by bosonic degrees of freedom with sizable couplings to the Higgs [4, 5].

Another motivation for extending the SM is the strong observational evidence for dark matter (DM), plausibly in the form of weakly interacting heavy particles. Even in the absence of a Higgs mass parameter in the UV, such particles will generically introduce additive corrections to the Higgs mass parameter and spoil the CW dynamics in the absence of additional symmetries. This motivates an alternative implementation of the CW mechanism, first proposed by Hempfling [6]. In this model, the Higgs couples to one extra scalar, which through dynamics of a hidden sector undergoes CW symmetry breaking and communicates the corresponding mass scale through the Higgs portal to the SM. Dark matter can then be given by any of the new hidden sector fields that govern the renormalization group evolution of the scalar potential in the dark sector.

There has been a lot of recent interest in models that implement various aspects of these basic ideas $[4,5,7-36]$. Here we will focus on implementations with dark sectors that are fairly simple and thus predictive. In section 2 we comment on issues of naturalness as applied to classically scale invariant modificiations of the SM, without claiming to resolve these issues. In section 3 we show, that in extensions of the SM with no explicit mass scales, the combination of a Higgs mass term generated through CW symmetry breaking together with the restriction to have a stable vacuum up to the Planck scale generically sets an upper bound on the dark matter mass scale of the order of a few TeV. Furthermore, 
the CW mechanism requires sizable couplings for gauge fields in the hidden sector, so that the simplest models in the literature are in addition subject to a lower bound on the DM mass of several hundred GeV. In section 4 we present a model with additional fermions in the hidden sector that can be dark matter candidates with masses at the electroweak scale or below. In sections 5, 6 and 7, we discuss the collider and dark matter phenomenology of the model. In section 7 , we also comment on further implications of this model for the dynamics of galaxy structure formation and a possible first order electroweak phase transition. We conclude in section 8.

The one loop effective potential of the discussed model and the one loop beta functions of the dark sector couplings are collected in appendices A and B. For the beta functions and anomalous dimensions, we follow the methods, conventions and notation of Machacek and Vaughn [37-39], with the improvements and extensions introduced by Luo and Xiao [40-42]. For the effective potentials, we follow the methods and conventions of Martin [43]. There are slight differences of notation in the literature: for example compared to [44, 45], our scalar self-coupling is twice as large, and our convention for anomalous dimensions has the opposite sign.

\section{Motivation}

A Coleman-Weinberg mechanism as the origin of electroweak symmetry breaking was first considered by Gildener and Weinberg [3]. In the absence of the Higgs mass term, the Lagrangian of the SM exhibits classical scale invariance that is softly broken by quantum effects - the well known scale anomaly. In UV completions of the SM, the physical thresholds associated with new massive states would constitute an explicit breaking of this symmetry. This introduces the need for a fine-tuning of the bare Higgs mass parameter against radiative corrections involving more massive particles. The fact that the Higgs mass parameter is not protected by a symmetry from these radiative corrections is known as the naturalness or hierarchy problem.

If the SM is UV completed by a conformal or supersymmetric (SUSY) theory, the Higgs mass parameter is radiatively stable above the scale at which this completion sets in; thus if this scale is not too high, the hierarchy problem is solved. This has led to the expectation that such a UV completion is realized in the vicinity of the electroweak scale. However, the new degrees of freedom predicted by either supersymmetric or conformal UV completions have not been observed, yet. This raises the prospect that the UV scale at which they set in is considerably higher than the electroweak scale, leaving the naturalness problem unresolved.

There are a number of experimental observations and theoretical questions, unrelated to the naturalness problem, that point to new high energy scales. Neutrino masses, gauge coupling unification, dark matter, and the expectation of a more fundamental theory of gravity are all expected to introduce new scales and as a consequence introduce an additive renormalization of the Higgs mass parameter. None of these arguments, however, necessarily points to a new UV scale relevant to the hierarchy problem. Neutrinos could be Dirac fermions with tiny Yukawa couplings, gauge coupling unification may not occur 
or may not imply new superheavy states, and dark matter could well be related to the electroweak scale itself.

There is still the challenging question of quantum gravity. We know that gravity does not make sense as a fundamental (perturbative) quantum field theory at short distances [46]. Naively, the Planck scale is expected to correspond at least roughly to a physical threshold where new massive states appear. In string theory this is indeed the case, and one can also argue that the existence of microscopic black holes is enough to require fine-tuning of the Higgs mass parameter [47]. A non-perturbative theory of quantum gravity might avoid this problem, but not if it resembles strongly-coupled gauge theories where new massive states are connected to the scale of strong coupling. At present no mechanism is known that can realize even a toy model for the type of UV completion that would avoid the hierachy problem, despite promising models in 2d [48].

On the other hand, all claims about Planckian physics and resulting effects on the renormalization of the Higgs mass parameter are, at this point, speculative. Generic UV completions of the Standard Model certainly have a Higgs naturalness problem [49], but for all we know, spacetime geometry breaks down at the Planck scale, and whether this results in a physical cut-off of relevance to the Higgs mass parameter is an open question.

Following the same line of reasoning, it is not clear to what extent the existence of ultra-Planckian Landau poles, as occurs for the hypercharge gauge coupling of the SM, should be regarded as a fundamental issue. In particular, our semi-classical understanding of gravity seems to indicate that such Landau poles are unobservable; the requisite scattering experiments would presumably be dominated by black hole production long before reaching the regime where incipient strong coupling in the hypercharge interactions would show itself. ${ }^{1}$ It is striking that no couplings of the SM run into a Landau pole below the Planck scale, which would be an unambigous sign of a new scale and therefore of the need (presumably) to fine-tune the Higgs mass.

In this paper, we assume that all explicit mass parameters vanish at the Planck scale, either as the consequence of the UV completed Planckian theory, or in spite of it. In addition we will focus on extensions of the SM that are weakly coupled and have no vacuum instability below the Planck scale.

\section{UV stability and IR instability from dark sectors}

\subsection{UV stability}

It is an intriguing observation about the Standard Model, that it seems to be consistent up to very high mass scales. Below the Planck scale, the only hint for New Physics within the SM itself is a possible instability of the electroweak vacuum triggered by the large top Yukawa. In the SM, the observed Higgs mass of $m_{h} \simeq 125.5 \mathrm{GeV}$ implies a Higgs quartic coupling at the electroweak scale of around $\lambda_{H}^{\mathrm{SM}}\left(m_{t}\right) \simeq 0.254[44,45]$. With this infrared boundary condition, assuming central values for $m_{t}$ and $\alpha_{s}$, the Higgs quartic coupling

\footnotetext{
${ }^{1}$ This observation is from Steve Giddings.
} 
runs negative at scales around $10^{10} \mathrm{GeV}$ and stays at a small negative value $\lambda_{H} \simeq-0.02$ up to the Planck scale, rendering the electroweak vacuum unstable $[44,45]{ }^{2}$

The instability can for example be overcome by the extension of the SM by a complex scalar $\Sigma$ with portal coupling to the Higgs $H$, so that the most general scalar potential reads

$$
V(H, \Sigma)=\mu_{H}^{2} H^{\dagger} H+\frac{\lambda_{H}}{2}\left(H^{\dagger} H\right)^{2}+\mu_{\Sigma}^{2} \Sigma^{\dagger} \Sigma+\frac{\lambda_{\Sigma}}{2}\left(\Sigma^{\dagger} \Sigma\right)^{2}+\lambda_{\Sigma H} \Sigma^{\dagger} \Sigma H^{\dagger} H .
$$

This potential is absolutely stable if the following condition is fulfilled

$$
\lambda_{H} \cos ^{4} \theta+\lambda_{\Sigma} \sin ^{4} \theta+2 \lambda_{\Sigma H} \sin ^{2} \theta \cos ^{2} \theta>0
$$

for all values of the angle $\theta$. In particular, this implies the necessary conditions

$$
\lambda_{H}>0, \quad \lambda_{\Sigma}>0, \quad \lambda_{H}+\lambda_{\Sigma}+2 \lambda_{\Sigma H}>0 .
$$

The new scalar can affect the stability of the Higgs potential in two ways: (i) by changing the beta function of the Higgs quartic; (ii) by changing the infrared boundary condition of the Higgs quartic. We briefly review both possibilities.

(i) The portal coupling $\lambda_{\Sigma H}$ gives a positive contribution to the beta function of the Higgs quartic. At the one loop level we have

$$
\begin{aligned}
& \beta_{\lambda_{H}}=\frac{1}{16 \pi^{2}}\left\{12 \lambda_{H}^{2}-\lambda_{H}\left(3\left(g^{\prime}\right)^{2}+9 g^{2}\right)+\frac{3}{4}\left(g^{\prime}\right)^{4}+\frac{3}{2}\left(g^{\prime}\right)^{2} g^{2}+\frac{9}{4} g^{4}\right. \\
& \left.+12 \lambda_{H} Y_{t}^{2}-12 Y_{t}^{4}+2 \lambda_{\Sigma H}^{2}\right\}
\end{aligned}
$$

where $g^{\prime}$ and $g$ are the $\mathrm{U}(1)$ and $\mathrm{SU}(2)$ gauge couplings, $Y_{t}$ is the top Yukawa coupling and we neglected the contributions from all other Yukawa couplings. In the SM, the top Yukawa contribution, $-12 Y_{t}^{4}$, dominates at low scales and drives the Higgs quartic coupling negative. If $\lambda_{\Sigma H}$ is sufficiently large, it can balance the top contribution and stabilize the vacuum. If in addition the vacuum expectation value of the new scalar vanishes, $\langle\Sigma\rangle=0$, the scalar can be stable and is a dark matter candidate [50-53].

(ii) If the new scalar has a non-vanishing vev, $\langle\Sigma\rangle=w / \sqrt{2}$, the tree level scalar mass matrix in the broken phase of (3.1) reads

$$
\mathcal{M}^{2}=\left(\begin{array}{cc}
\lambda_{H} v^{2} & \lambda_{\Sigma H} w v \\
\lambda_{\Sigma H} w v & \lambda_{\Sigma} w^{2}
\end{array}\right) .
$$

In the limit $\lambda_{\Sigma} w^{2} \gg \lambda_{H} v^{2}$, the light Higgs-like mass eigenstate has a mass

$$
m_{h}^{2}=\left(\lambda_{H}-\frac{\lambda_{\Sigma H}^{2}}{\lambda_{\Sigma}}\right) v^{2}+\mathcal{O}\left(\frac{v^{4}}{w^{2}}\right) .
$$

In order to reproduce a Higgs mass of $125.5 \mathrm{GeV}$, the value of $\lambda_{H}$ at the electroweak scale has to be larger than in the SM. In that way the UV instability can be avoided [54-56].

\footnotetext{
${ }^{2}$ The electroweak vacuum in the SM is still meta-stable, i.e. its lifetime is larger than the age of the universe.
} 
Indeed, for a Higgs quartic that is about $7 \%$ larger than in the SM, $\lambda_{H}\left(m_{t}\right) \simeq 0.273$, the central value of the Higgs quartic remains positive up to high scales and vanishes around the Planck mass. Taking into account uncertainties in the running of $\lambda_{H}$ from the top mass and strong gauge coupling, a positive Higgs quartic at the $2 \sigma$ level corresponds to

$$
0.259 \lesssim \lambda_{H}\left(m_{t}\right) \lesssim 0.288
$$

Interestingly enough, for such a range of boundary conditions not only the Higgs quartic, but also its beta function become zero at scales close to the Planck scale. The required size of the portal coupling to stabilize the potential in the UV is considerably smaller than using mechanism (i). If the scalar quartic $\lambda_{\Sigma}$ is of the same order of the Higgs quartic $\lambda_{H}$, a portal coupling of $\left|\lambda_{\Sigma H}\right| \sim 0.05$ is sufficient. Although the heavy scalar is unstable in scenario (ii), additional fields which get their mass from couplings to $\Sigma$ could explain dark matter.

As pointed out in [54], the correction in (3.6) from the heavy scalar persists even in the decoupling limit, $w \rightarrow \infty$, so that neither mechanism to mitigate the vacuum instability clearly points to a specific scale for the extra sector. ${ }^{3}$ This situation is fundamentally different in models with classical scale invariance in the UV. If the Higgs mass parameter $\mu_{H}^{2}$ in (3.1) is zero it will be generated by the vev of the extra scalar through the portal coupling

$$
\lambda_{\Sigma H} \Sigma^{\dagger} \Sigma H^{\dagger} H \quad \rightarrow \quad \frac{\lambda_{\Sigma H} w^{2}}{2} H^{\dagger} H .
$$

In that case, the ratio of vacuum expectation values is controlled by the portal coupling (note that $\lambda_{\Sigma H}$ has to be negative to trigger a vev for the Higgs boson)

$$
\frac{v^{2}}{w^{2}}=-\frac{\lambda_{\Sigma H}}{\lambda_{H}}
$$

and the correction in (3.6) does decouple for $w \rightarrow \infty$. For a vanishing Higgs mass parameter $\mu_{H}^{2}=0$ and for the Higgs being the lightest scalar, the vev of the extra scalar is therefore bounded from above. The requirement that the central value ( $2 \sigma$ upper bound) of $\lambda_{H}$ remains positive up to the Planck scale implies

$$
w \lesssim\left(\lambda_{\Sigma}\right)^{-\frac{1}{4}} \times 350(470) \mathrm{GeV}
$$

Here, we worked in the limit $\lambda_{\Sigma} w^{2} \gg \lambda_{H} v^{2}$ and neglect the tiny $\lambda_{\Sigma H}$ contributions to the running of $\lambda_{H}$. In the limit in which $\lambda_{\Sigma} w^{2}=\lambda_{H} v^{2}$ the bounds become $w \lesssim 3.5(12.7) \mathrm{TeV}$. This corresponds to the extreme case of maximal mixing between the Higgs and the dark scalar. As we will discuss in section 5, the mixing is strongly constrained by collider bounds.

\subsection{IR instability}

We now address the question of how to generate the vev for the scalar $\Sigma$. In particular, if not only the Higgs mass parameter, but all scales in the potential (3.1) vanish in the UV,

\footnotetext{
${ }^{3} \mathrm{In}$ both mechanisms however the scale has to be at least somewhat below $10^{10} \mathrm{GeV}$, the scale where the Higgs quartic crosses zero.
} 
$\mu_{H}^{2}=\mu_{\Sigma}^{2}=0$, the vev of the extra scalar can only be induced radiatively, either through strong dynamics, in which a new condensation scale induces a mass term for the extra scalar $[16,25,57]$, or by a Coleman-Weinberg mechanism, in which the balance between the quartic and the one-loop corrections to the effective potential determine the vev [2]. We will concentrate on the latter mechanism in the following.

In the limit of small Higgs portal coupling $\left|\lambda_{\Sigma H}\right| \ll 1$, we can consider the effective potential $V_{\text {eff }}$ for the scalar independently from the Higgs boson. Its general one loop form is given by

$$
V_{\mathrm{eff}}(s, \mu)=\frac{1}{8} \lambda_{\Sigma}(\mu) s^{4}+\frac{B(\mu)}{4} s^{4} \log \left(\frac{s^{2}}{\mu^{2}}\right)
$$

in which the scalar component $s$ in (4.4) is treated as a background field and $\mu$ is the renormalization scale. Subleading terms in $V_{\text {eff }}$ that are proportional to the anomalous dimension of $s$ are suppressed. This effective potential has a local minimum if

$$
\left.\frac{\partial V_{\mathrm{eff}}(s, \mu)}{\partial s}\right|_{\mu=s=w}=0 \quad \Rightarrow \quad B=-\lambda_{\Sigma}
$$

and

$$
\left.\frac{\partial^{2} V_{\mathrm{eff}}(s, \mu)}{\partial s^{2}}\right|_{\mu=s=w}>0 \quad \Rightarrow \quad B>0
$$

Since $B$ is a loop-suppressed function, it follows, that in the vicinity of the minimum, the quartic coupling needs to be small and negative for CW symmetry breaking to work. For the full potential, including terms proportional to the portal coupling, $\lambda_{\Sigma H}$, the condition $\lambda_{\Sigma}<0$ is replaced by [58]

$$
4 \lambda_{H} \lambda_{\Sigma}-\lambda_{\Sigma H}^{2}<0 \text {. }
$$

As long as the portal coupling is small, $\lambda_{\Sigma H}^{2} \ll \beta_{\lambda_{\Sigma}}^{(1)} \lambda_{H}$, this gives approximately the same constraint. ${ }^{4}$ Further, the coefficient $B$ is related to the beta-function of $\lambda_{\Sigma}$ by the one-loop renormalization group equation

$$
\mu \frac{\partial}{\partial \mu} V_{1}(s, \mu)+\beta_{\lambda_{\Sigma}}^{(1)} \frac{d}{d \lambda_{\Sigma}} V_{0}-\gamma_{s}^{(1)} s \frac{d}{d s} V_{0}=0,
$$

in which $\gamma_{s}^{(1)}$ denotes the one-loop anomalous dimension of the scalar fields $s$ and $\beta_{\lambda_{\Sigma}}^{(1)}$ the one-loop beta function of the quartic coupling. From the general form (3.11) follows

$$
\beta_{\lambda_{\Sigma}}^{(1)}=4 \gamma_{s}^{(1)} \lambda_{\Sigma}+4 B
$$

Close to the minimum, the first term can be neglected to good approximation. Therefore the beta function of $\lambda_{\Sigma}$ has to be positive to induce a vev for the scalar. A natural way to ensure a positive beta function in a region around the minimum is to charge the scalar under a dark gauge symmetry. Loops with dark gauge bosons give a positive contribution to $\beta_{\lambda_{\Sigma}}$ and lead to the desired IR instability of the scalar potential. At the same time, a positive beta function for the quartic coupling ensures that the scalar potential is stable in the UV.

\footnotetext{
${ }^{4}$ This situation changes for models in which the two-loop contribution becomes relevant [5].
} 


\subsection{The scale of dark matter}

Independent on whether the vev of the dark scalar is induced by strong dynamics or by a Coleman-Weinberg mechanism as discussed in the previous section, additional fields with couplings to $\Sigma$ are required, which can provide dark matter candidates. If the new sector does not introduce explicit mass scales, the masses of any new states can only be generated through the vev of the extra scalar. In this context it is very interesting that the range suggested by stability considerations seems to agree with the mass scales suggested by the "WIMP miracle". We emphasize that this is a generic feature of extensions of the SM with the above properties. Extended models with additional scalars can of course soften this relation.

Examples of models in the literature, in which the electroweak vacuum is stabilized through the Higgs portal and dimensionful couplings are absent, reveal the mentioned connection between the dynamical generation of a vacuum expectation value in the IR, the stabilization of the vacuum in the UV, and the dark matter sector: the authors of [13] discuss a model with an extra scalar charged under a $\mathrm{U}(1)_{\mathrm{B}-\mathrm{L}}$ that gives a Majorana mass to right-handed neutrinos through a CW mechanism. The $Z^{\prime}$ in this model is not a candidate for DM, because it couples to $B-L$ and the resulting experimental bounds on the $Z^{\prime}$ push the vev of the extra scalar above $3 \mathrm{TeV}$. As a consequence, the vacuum cannot be stabilized up to the Planck scale in this model. In [17], the extra scalar is a doublet under an additional dark $\mathrm{SU}(2)$ and breaks it completely. In this case the heavy gauge boson triplet constitutes dark matter, and if vacuum stability is enforced, the vev of the scalar is bound to be at the $\mathrm{TeV}$ scale. In addition, the authors of [28] have shown, that the extra gauge couplings that drive the quartic of the extra scalar negative in the IR need to be of order one in these models in order to stabilize the vacuum, so that the masses of the corresponding gauge bosons are bound from below by $m=g w / 2 \gtrsim 500 \mathrm{GeV}$.

If the hidden sector in addition to scalars and gauge bosons has also fermionic degrees of freedom, they are generically required to be lighter than the gauge bosons. This can easily be understood from the fact that they enter the effective potential with a negative sign,

$$
V_{\text {eff }}(s, \mu)=V_{0}(s, \mu)+\frac{1}{64 \pi^{2}} \sum_{i=B, F} n_{i} m_{i}^{4}(s)\left[\log \frac{m_{i}^{2}(s)}{\mu^{2}}-C_{i}\right],
$$

where the sum goes over fermions (F) and bosons (B), $m_{i}(s)$ denotes the corresponding Higgs dependent masses, $n_{i}=\mp$ the number of fermionic/bosonic degrees of freedom, and the $C_{i}$ are renormalization scheme dependent constants. If the fermionic contributions dominate the one loop contributions to the effective potential (3.17), the condition (3.13) cannot be fulfilled. Hence, the effective potential is unbounded from below, i.e. the fermions generate a UV instability instead of a IR instability. Therefore, for the CW mechanism to work, the gauge bosons are generically heavier and fermions constitute dark matter. In the following sections we will discuss in detail an example of a model

- that does not contain any explicit mass scales,

- that utilizes the Coleman-Weinberg mechanism in a dark sector to induce spontaneous electroweak symmetry breaking through a Higgs portal, 
- that is weakly coupled below the Planck scale, with all of the running scalar quartic couplings starting near zero at the Planck scale,

- that stabilizes the vacuum until the Planck scale, and

- that contains fermionic dark matter with masses at or below the electroweak scale.

\section{The model}

We consider an extension of the $\mathrm{SM}$ by a $\mathrm{SU}(2)_{X} \times \mathrm{U}(1)_{X}$ gauge group, under which all SM fields are uncharged. In addition to the $\mathrm{SU}(2)_{X} \times \mathrm{U}(1)_{X}$ gauge bosons $W_{a}^{\prime}$ and $B^{\prime}$, we introduce a scalar doublet $\Sigma$ under $\mathrm{SU}(2)_{X}$ with $\mathrm{U}(1)_{X}$ charge $Q_{\Sigma}^{X}=1 / 2$. The fermionic sector consists of two sets of chiral SM singlet fermions: $\psi_{i}^{L}, \xi_{i}^{R}, \chi_{i}^{R}$, with $i=1,2$. The left handed fields $\psi_{1}^{L}=\left(\chi_{1}^{L}, \xi_{1}^{L}\right)$ and $\psi_{2}^{L}=\left(\xi_{2}^{L}, \chi_{2}^{L}\right)$ are $\mathrm{SU}(2)_{X}$ doublets, while the right handed ones are $\mathrm{SU}(2)_{X}$ singlets. We assign the following dark hypercharges that ensure anomaly cancellation: $Q_{\psi_{1}}^{X}=+1 / 2, Q_{\psi_{2}}^{X}=-1 / 2, Q_{\chi_{1}}^{X}=+1, Q_{\chi_{2}}^{X}=-1, Q_{\xi_{1}}^{X}=Q_{\xi_{2}}^{X}=0$.

We denote the field strength tensors of the $\mathrm{SU}(2)_{X}$ and $\mathrm{U}(1)_{X}$ gauge symmetries by $\left(W_{a}^{\prime}\right)_{\mu \nu}$ and $\left(B^{\prime}\right)^{\mu \nu}$, so that their kinetic terms read

$$
\mathcal{L}_{\text {gauge }}=\frac{1}{4}\left(W_{a}^{\prime}\right)_{\mu \nu}\left(W_{a}^{\prime}\right)^{\mu \nu}+\frac{1}{4}\left(B^{\prime}\right)_{\mu \nu}\left(B^{\prime}\right)^{\mu \nu},
$$

where $a=1,2,3$ is the index of the adjoint of $\mathrm{SU}(2)_{X}$. We assume that there is no kinetic mixing between the $\mathrm{U}(1)_{X}$ gauge boson and the SM hypercharge gauge boson. As our model does not contain fields that are charged under both $\mathrm{U}(1)$ symmetries, such a choice is stable under radiative corrections. In the absence of kinetic U(1) mixing and not considering the dark singlet fermions, the only renormalizable portal between the dark sector and the SM is the mixing of the dark scalar with the Higgs. Explicit mass terms for the scalars are assumed to vanish, such that

$$
\mathcal{L}_{\text {scalar }}=|D H|^{2}+|D \Sigma|^{2}-\frac{\lambda_{H}}{2}|H|^{4}-\frac{\lambda_{\Sigma}}{2}|\Sigma|^{4}-\lambda_{\Sigma H}|H|^{2}|\Sigma|^{2} .
$$

The covariant derivatives of $H$ and $\Sigma$ are given by (Lorentz indices are suppressed for simplicity)

$$
D H=\left(\partial-i \frac{g}{2} \sigma^{a} W_{a}-i g^{\prime} Q_{H} B\right) H, \quad D \Sigma=\left(\partial-i \frac{g_{X}}{2} \sigma^{a} W_{a}^{\prime}-i g_{X}^{\prime} Q_{\Sigma}^{X} B^{\prime}\right) \Sigma .
$$

Here, $g$ and $g^{\prime}$ are the $\mathrm{SU}(2)$ and $\mathrm{U}(1)$ gauge couplings of the SM, and $g_{X}$ and $g_{X}^{\prime}$ are the corresponding couplings in the dark sector. The Higgs and the dark scalar can be decomposed as follows

$$
H=\left(\begin{array}{c}
G^{+} \\
\frac{1}{\sqrt{2}}\left(h+v+i G^{0}\right)
\end{array}\right), \quad \Sigma=\left(\begin{array}{c}
a^{+} \\
\frac{1}{\sqrt{2}}(s+w+i a)
\end{array}\right),
$$

where $v(w)$ is the respective vacuum expectation value that breaks the $\mathrm{SU}(2)_{(X)} \times \mathrm{U}(1)_{(X)}$ gauge group down to (dark) electromagnetism. The Goldstone bosons $G^{ \pm}, G^{0}$ and $a^{ \pm}, a$ 
provide the longitudinal components of the $W$ and $Z$ boson of the SM, as well as the corresponding $W^{\prime}$ and $Z^{\prime}$ in the dark sector. The masses of the dark gauge bosons are given by

$$
m_{\gamma^{\prime}}=0, \quad m_{W^{\prime}}=\frac{w}{2} g_{X}, \quad m_{Z^{\prime}}=\frac{w}{2} \sqrt{g_{X}^{2}+g_{X}^{\prime 2}} .
$$

Analogous to the photon in the SM, the dark sector contains a massless gauge boson, which we will refer to as dark photon, $\gamma^{\prime}$. In complete analogy to the SM, we define a dark electromagnetic coupling $e_{X}$ as well as a dark mixing angle $\theta_{X}$

$$
e_{X}=\frac{g_{X} g_{X}^{\prime}}{\sqrt{g_{X}^{2}+g_{X}^{\prime 2}}}, \quad c_{X}=\cos \theta_{X}=\frac{g_{X}}{\sqrt{g_{X}^{2}+g_{X}^{\prime 2}}}, \quad s_{X}=\sin \theta_{X}=\frac{g_{X}^{\prime}}{\sqrt{g_{X}^{2}+g_{X}^{\prime 2}}} .
$$

The dark fermions couple to the extra scalar $\Sigma$ through Yukawa couplings,

$$
\begin{aligned}
\mathcal{L}_{\text {fermion }}= & i \bar{\psi}_{i}^{L} \not D \psi_{i}^{L}+i \bar{\chi}_{i}^{R} \not D \chi_{i}^{R}+i \bar{\xi}_{i}^{R} \not \partial \xi_{i}^{R} \\
& +\left(Y_{\chi_{1}} \bar{\psi}_{1}^{L} \chi_{1}^{R} \tilde{\Sigma}+Y_{\chi_{2}} \bar{\psi}_{2}^{L} \chi_{2}^{R} \Sigma+Y_{\xi_{1}} \bar{\psi}_{1}^{L} \xi_{1}^{R} \Sigma+Y_{\xi_{2}} \bar{\psi}_{2}^{L} \xi_{2}^{R} \tilde{\Sigma}+\text { h.c. }\right),
\end{aligned}
$$

where $\tilde{\Sigma}=i \sigma_{2} \Sigma^{*}$.

As in the scalar sector, we do not consider explicit Majorana mass terms for the fermions that would be allowed given the quantum number assignments for $\psi_{i}, \chi_{i}$, and $\xi_{i}$. Moreover, we do not allow Yukawa couplings of the dark singlets $\xi_{i}^{R}$ with the SM lepton doublets. The absence of Majorana masses, flavor off-diagonal Yukawa couplings and lepton Yukawa couplings can for example be enforced by demanding dark fermion number conservation. The covariant derivatives of the fermions are

$$
D \psi_{i}^{L}=\left(\partial-i \frac{g_{X}}{2} \sigma^{a} W_{a}^{\prime}-i g_{X}^{\prime} Q_{\psi_{i}}^{X} B^{\prime}\right) \psi_{i}^{L}, \quad D \chi_{i}^{R}=\left(\partial-i g_{X}^{\prime} Q_{\chi_{i}}^{X} B^{\prime}\right) \chi_{i}^{R},
$$

and $\xi_{i}^{R}$ are total singlets. After breaking of the dark $\mathrm{SU}(2)_{X} \times \mathrm{U}(1)_{X}$ by the vev of $\Sigma$, the fermions become massive and we introduce the Dirac spinors $\chi_{i}=P_{L} \chi_{i}+P_{R} \chi_{i}=\left(\chi_{i}^{L}, \chi_{i}^{R}\right)$ and $\xi_{i}=P_{L} \xi_{i}+P_{R} \xi_{i}=\left(\xi_{i}^{L}, \xi_{i}^{R}\right)$ with masses

$$
m_{\chi_{i}}=\frac{Y_{\chi_{i}}}{\sqrt{2}} w, \quad m_{\xi_{i}}=\frac{Y_{\xi_{i}}}{\sqrt{2}} w
$$

Conservation of dark fermion number and dark electromagnetism implies that both $\chi_{i}$ and $\xi_{i}$ can be stable dark matter candidates.

\subsection{The scalar spectrum}

The one loop effective potential of the model is given in the appendix A. If the bosonic contributions to the effective potential dominate over the fermionic ones, non-zero scalar vevs will be induced radiatively. In the limit of a small portal coupling, the vevs of the Higgs $v$ and of the dark scalar $w$ are approximately connected by the relation (3.9). Neglecting the effects of the field anomalous dimensions of the Higgs and the dark scalar as well as the running of both the SM quartic coupling and the portal coupling, while keeping the 
dominant contribution from the running of $\lambda_{\Sigma}$, the scalar mass matrix in the minimum of the potential can be written as

$$
\mathcal{M}^{2} \simeq \frac{v^{2}}{2}\left(\begin{array}{cc}
2 \lambda_{H} & -2 \sqrt{\lambda_{H}\left|\lambda_{\Sigma H}\right|} \\
-2 \sqrt{\lambda_{H}\left|\lambda_{\Sigma H}\right|} & 2\left|\lambda_{\Sigma H}\right|+\lambda_{H} \beta_{\lambda_{\Sigma}} /\left|\lambda_{\Sigma H}\right|
\end{array}\right)
$$

This mass matrix can be diagonalized through the rotation

$$
\left(\begin{array}{l}
h \\
s
\end{array}\right) \rightarrow\left(\begin{array}{cc}
c_{\alpha} & s_{\alpha} \\
-s_{\alpha} & c_{\alpha}
\end{array}\right)\left(\begin{array}{l}
h \\
s
\end{array}\right), \quad \sin 2 \alpha=\frac{2 \sqrt{\lambda_{H}\left|\lambda_{\Sigma H}\right|} v^{2}}{m_{s}^{2}-m_{h}^{2}}
$$

with $s_{\alpha}=\sin \alpha$ and $c_{\alpha}=\cos \alpha$. The mass eigenvalues $m_{h}$ and $m_{s}$ are given by

$$
m_{h}^{2} \simeq v^{2}\left(\lambda_{H}-\frac{2 \lambda_{\Sigma H}^{2}}{\beta_{\lambda_{\Sigma}}-2\left|\lambda_{\Sigma H}\right|}\right), \quad m_{s}^{2} \simeq v^{2}\left(\frac{\lambda_{H} \beta_{\lambda_{\Sigma}}}{2\left|\lambda_{\Sigma H}\right|}+\frac{\beta_{\lambda_{\Sigma}}\left|\lambda_{\Sigma H}\right|}{\beta_{\lambda_{\Sigma}}-2\left|\lambda_{\Sigma H}\right|}\right),
$$

where we expanded to first order in the limit $\lambda_{\Sigma H}, \beta_{\lambda_{\Sigma}} \ll \lambda_{H}$. In this limit, the mass of the dark scalar is directly proportional to the beta function of the dark scalar quartic coupling. If the dark scalar beta function is larger than twice the absolute value of the portal coupling,

$$
\beta_{\lambda_{\Sigma}} \gtrsim 2\left|\lambda_{\Sigma H}\right|
$$

the dark scalar is heavier than the Higgs boson, and the mass of the Higgs boson is reduced compared to the Standard Model expression.

\subsection{Vacuum stability in the UV}

We now discuss the renormalization group running of the model parameters up to high scales and demonstrate that the electroweak minimum in the scalar potential can be absolutely stable. The one loop beta functions for all couplings of the dark sector as well as the one loop correction to the beta function of the Higgs quartic are collected in the appendix B. For the SM beta functions we use 2 loop results from [37-42].

As discussed already in section 3 and as shown in Equation (4.12), the physical Higgs mass is not completely determined by the Higgs quartic, but gets an additional contribution from the mixing with the dark scalar. If the dark scalar is heavier than the Higgs, mixing effects will reduce the Higgs mass and a quartic coupling larger than in the SM is required to accommodate a Higgs mass of $m_{h} \simeq 125.5 \mathrm{GeV}$. If the portal coupling is large enough, the IR boundary condition for $\lambda_{H}$ is such that $\lambda_{H}$ stays positive all the way to the Planck scale, or, in the limiting case, "touches" zero close to the Planck scale. The region of the parameter space where this can be achieved is shown in figure 1 in the plane of the scalar mass $m_{s}$ and the mixing angle $\sin \alpha$. In the shaded region the Higgs quartic is positive up to the Planck scale. Between the two dashed curves the limiting case where $\lambda_{H}$ touches zero close to the Planck scale can be realized within $2 \sigma$. The dotted lines in the unstable region indicate the scale in $\mathrm{GeV}$ where the Higgs quartic runs negative. The solid lines show contours of constant scalar vev $w$, that corresponds to a given scalar mass $m_{s}$ and mixing angle $\sin \alpha$. 


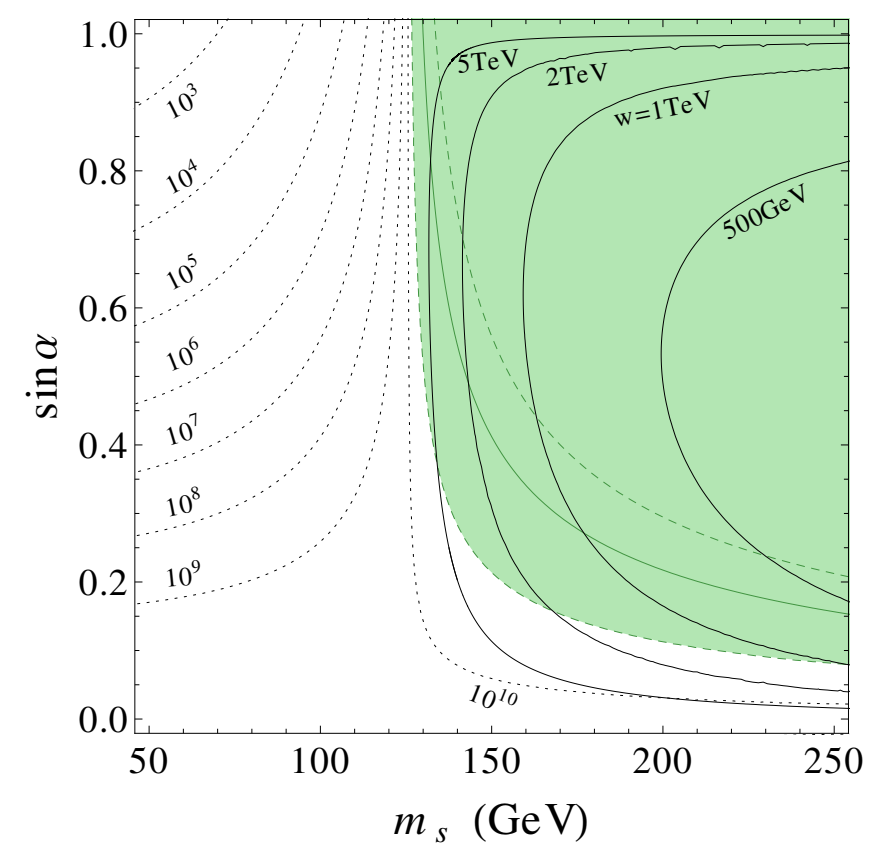

Figure 1. Vacuum stability properties in the $m_{s}-\sin \alpha$ plane. In the shaded region the Higgs quartic is positive up to the Planck scale. Between the two dashed contours the Higgs quartic touches zero close to the Planck scale within $2 \sigma$. The dotted lines in the unstable region show the scale at which the Higgs quartic runs negative. The solid lines indicate contours of constant scalar vev, $w$. Note, that large mixing angles $\sin \alpha \gtrsim 0.5$ are phenomenologically strongly constrained by collider bounds, see section 5 .

As we will discuss in section 5 , the mixing angle is bounded at the order of $\sin \alpha \lesssim$ 0.5. This implies a typical value for $w$ around the $\mathrm{TeV}$ scale, and an upper bound of several $\mathrm{TeV}$, as expected from the general discussion in section 3. On the other hand, scalar vevs considerably below a $\mathrm{TeV}$ can in principle be achieved by increasing $\left|\lambda_{\Sigma H}\right|$ (see Equation (3.9)). However, this requires that the beta function $\beta_{\lambda_{\Sigma}}$ needs to to be increased simultaneously due to the bound (4.13). The beta function $\beta_{\lambda_{\Sigma}}$ is also bounded from above by perturbativity requirements on the dark gauge couplings. As a result, values for $w$ considerably below the $\mathrm{TeV}$ scale are disfavored. In the following, we will concentrate on regions of parameter space with $w=\mathcal{O}(1 \mathrm{TeV})$ and a Higgs quartic that touches zero close to the Planck scale.

As long as the dark fermion Yukawa couplings are not too large, the beta function of the scalar quartic $\beta_{\lambda_{\Sigma}}$ is dominated by the dark gauge couplings and stays positive. In such a case, $\lambda_{\Sigma}$ increases monotonically with the RG scale and is always positive in the UV. Note, however, that sizable dark fermion Yukawas can modify the behavior of $\lambda_{\Sigma}$ in the UV. In particular, the model allows to accommodate the limiting case where not only the Higgs quartic but also the dark scalar quartic touches zero close to the Planck scale. In the approximation $\beta_{\lambda_{\Sigma}} \approx 4 B$, it is straightforward to compute the leading terms in the beta function of the scalar quartic from comparing (3.17) with (3.11), using (4.5) and (4.9),

$$
\beta_{\lambda_{\Sigma}}^{(1)} \approx \frac{1}{16 \pi^{2}}\left\{\frac{9}{4} g_{X}^{4}+\frac{3}{2} g_{X}^{2} g_{X}^{\prime 2}+\frac{3}{4} g_{X}^{\prime 4}-4\left(Y_{\chi_{1}}^{4}+Y_{\chi_{2}}^{4}+Y_{\xi_{1}}^{4}+Y_{\xi_{2}}^{4}\right)\right\}
$$


The full one loop expression for the beta function can be found in appendix B. The beta function of $\lambda_{\Sigma}$ receives contributions dominantly from three sources: (i) from $\mathrm{SU}(2)_{X}$ gauge boson loops, (ii) from fermion loops, and (iii) from $\mathrm{U}(1)_{X}$ gauge boson loops. The gauge boson (fermion) loops increase (decrease) $\lambda_{\Sigma}$ for higher scales. At low scales, the $\mathrm{SU}(2)_{X}$ contribution dominates and leads to the infrared instability in the scalar potential as discussed above. With the given particle content, the $\mathrm{SU}(2)_{X}$ gauge interactions are asymptotically free. Therefore the contribution of the $\mathrm{SU}(2)_{X}$ gauge bosons to the running of the scalar quartic becomes smaller and smaller for higher scales. At sufficiently high scales, the dominant contributions to the beta function can come from the fermion Yukawa couplings, and the scalar quartic will start to decrease again. Finally, at scales close to the Planck scale, the $\mathrm{U}(1)_{X}$ gauge coupling, having a positive beta function, can become large and compensate the effect of the Yukawa couplings. It is possible to adjust parameters such that the scalar quartic as well as its beta function vanish exactly at the Planck scale.

The plots of figure 2 show the renormalization group evolution of the gauge couplings, Yukawa couplings and the scalar quartic couplings for an example parameter point of the model where such a limiting case is realized. ${ }^{5}$ The shown couplings correspond approximately to the following dark sector spectrum

$$
\begin{aligned}
& m_{h} \simeq 125.5 \mathrm{GeV}, \quad m_{s} \simeq 168 \mathrm{GeV}, \quad m_{W^{\prime}} \simeq 740 \mathrm{GeV}, m_{Z^{\prime}} \simeq 850 \mathrm{GeV}, \\
& m_{\chi_{1}} \simeq 50 \mathrm{GeV}, \quad m_{\chi_{2}} \simeq 50 \mathrm{GeV}, \quad m_{\xi_{1}} \simeq 160 \mathrm{GeV}, \quad m_{\xi_{2}} \simeq 700 \mathrm{GeV} .
\end{aligned}
$$

The values for the dark vev is

$$
w \simeq 1.1 \mathrm{TeV},
$$

and the masses in (4.15) (apart from the Higgs mass) correspond to running $\overline{\mathrm{MS}}$ masses at the scale $\mu=w$. The $\mathrm{SU}(2)_{X}$ gauge coupling is $\mathcal{O}(1)$ at the low scale, while the $\mathrm{U}(1)_{X}$ coupling is $\mathcal{O}(1)$ close to the Planck scale. The $\mathrm{U}(1)_{X}$ gauge coupling develops a Landau pole at around $10^{30} \mathrm{GeV}$, well above the Planck scale. Both the Higgs quartic $\lambda_{H}$ and the dark scalar quartic $\lambda_{\Sigma}$ as well as their beta functions are approximately 0 at the Planck scale. The portal coupling $\lambda_{\Sigma H}$ is small and negative at all scales but cannot run to zero at the Planck scale. It is the only link between the SM and the dark sector and is therefore only multiplicatively renormalized.

\section{$5 \quad$ Higgs and dark scalar phenomenology}

The considered model leads to various testable predictions for Higgs phenomenology. Due to the mixing of the two scalars, the couplings of the Higgs boson $h$ to all SM particles are suppressed by a factor $c_{\alpha}$ compared to the SM case, resulting in an overall suppression of all Higgs rates by $c_{\alpha}^{2}$. The latest results from Higgs rate measurements from ATLAS [59] and CMS [60] read

$$
\mu_{\mathrm{ATLAS}}=1.30_{-0.17}^{+0.18}, \quad \mu_{\mathrm{CMS}}=1.00 \pm 0.09_{-0.07}^{+0.08} \pm 0.07,
$$

\footnotetext{
${ }^{5}$ Note that due to the small negative portal coupling $\lambda_{\Sigma H}$, the vacuum is actually not absolutely stable in the shown example. Absolute stability requires that $\lambda_{H}$ and $\lambda_{\Sigma}$ are at least of the same size as the (tiny) absolute value of the portal coupling, see (3.3).
} 

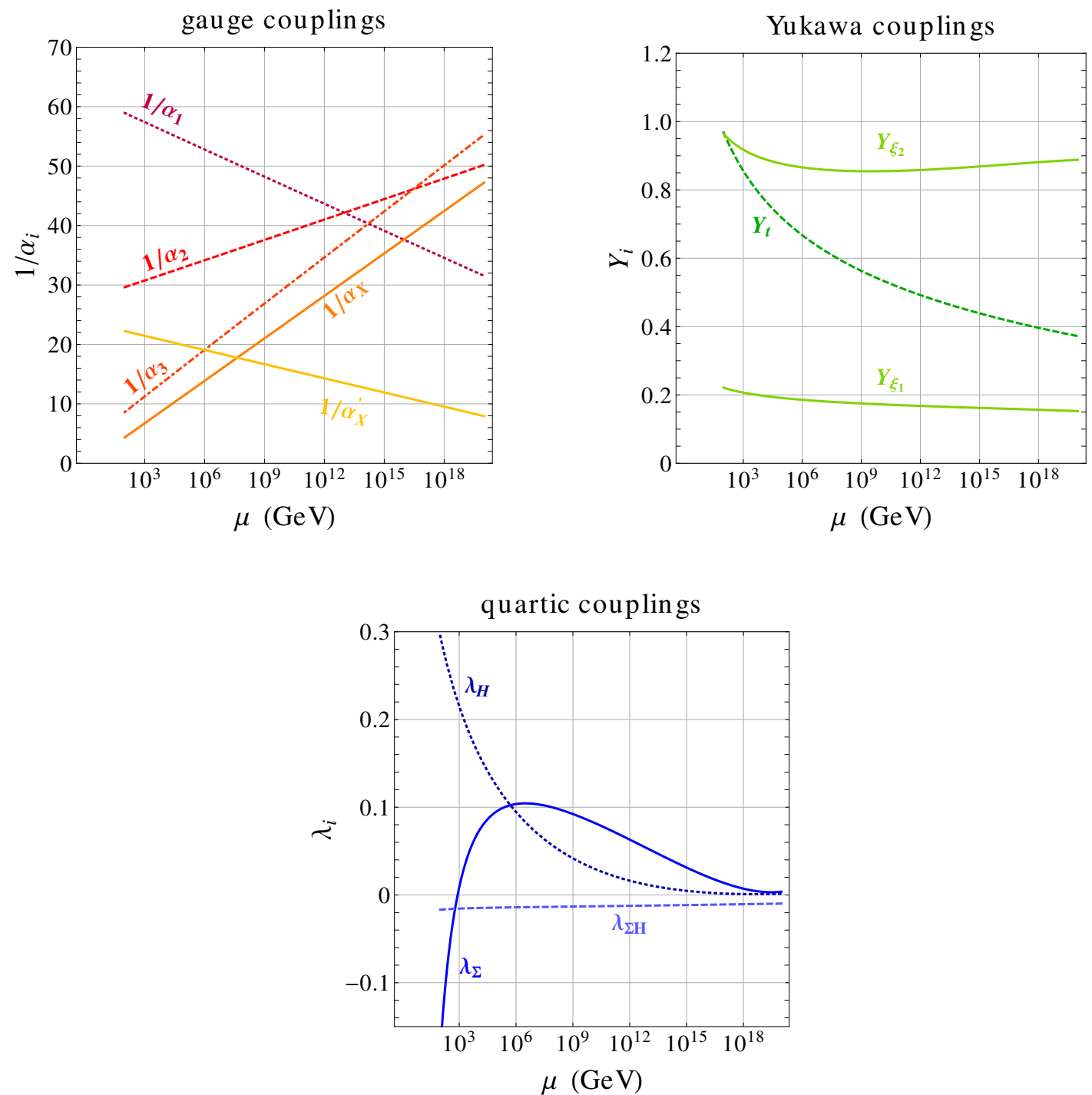

Figure 2. The renormalization group evolution of the gauge couplings, the Yukawa couplings and the scalar quartic couplings for one example parameter point in the considered model that leads to an almost flat scalar potential at the Planck scale.

which we will interpret roughly as a constraint of $c_{\alpha} \gtrsim 0.9$, equivalent to a $20 \%$ reduction of the SM production rate. At the next run of the LHC, the precision of the rate measurements is expected to be improved by around a factor of 3, which will allow to probe deviations of $c_{\alpha}$ from unity of the order of $5 \%$.

Moreover, the mixing of the Higgs with the dark scalar also leads to couplings of $h$ to the fermions in the dark sector. If some of these fermions are sufficiently light, the Higgs can decay into them. We find for the corresponding partial decay widths

$$
\Gamma\left(h \rightarrow f_{i} f_{i}\right)=\frac{Y_{f_{i}}^{2}}{8 \pi} m_{h} s_{\alpha}^{2}\left(1-\frac{4 m_{f_{i}}^{2}}{m_{h}^{2}}\right)^{\frac{3}{2}},
$$


which applies for dark-charged and neutral fermions $f_{i}=\chi_{i}, \xi_{i}$. Analogous to the SM decay of the Higgs into two photons, the Higgs can also decay into two dark photons through loops of dark-charged fermions $\chi_{i}$ and the dark $W^{\prime}$ boson. In the limit $m_{W^{\prime}} \gg m_{h}$, we find for the $h \rightarrow \gamma^{\prime} \gamma^{\prime}$ decay width

$$
\Gamma\left(h \rightarrow \gamma^{\prime} \gamma^{\prime}\right) \simeq \frac{1}{16 \pi} s_{\alpha}^{2} \frac{m_{h}^{3}}{w^{2}}\left(\frac{g_{X}^{2}}{16 \pi^{2}}\right)^{2}\left|7-\sum_{i} \frac{8 m_{\chi_{i}}^{2}}{m_{h}^{2}}\left[1+\left(1-\frac{4 m_{\chi_{i}}^{2}}{m_{h}^{2}}\right) f\left(\frac{m_{h}^{2}}{4 m_{\chi_{i}}^{2}}\right)\right]\right|^{2} .
$$

The loop function $f$ is given in the appendix C. Given that $h \rightarrow \gamma^{\prime} \gamma^{\prime}$ is loop suppressed, it can only compete with the decay into dark fermions if the dark gauge coupling is large $g_{X} \gtrsim 1$ and the fermion Yukawas are very small, $Y_{\chi_{i}} \lesssim 10^{-2}$.

Given the tiny total width of the SM Higgs, $\Gamma_{h}^{S M} \simeq 4 \mathrm{MeV}$, even for moderate mixing angles $s_{\alpha}$ the induced invisible branching ratio can be sizable. This is illustrated in the upper plot of figure 3, that shows for various mixing angles $s_{\alpha}$ the branching ratio of the Higgs into the charged dark fermions as a function of the charged dark fermion mass, for the example choice $m_{\chi_{1}}=m_{\chi_{2}} / 2$. The dark vev is set to $w=1.5 \mathrm{TeV}$ and the neutral fermions are assumed to be heavier than at least half the Higgs mass. We observe that for moderate mixing angles of $s_{\alpha} \sim 0.3$, branching ratios into dark fermions of $\mathcal{O}(10 \%)$ are possible. The branching ratio can be even larger for smaller $w$. The branching ratio into dark photons is at most at the percent level and therefore hardly relevant. ATLAS and CMS search for invisible decays of Higgs bosons that are produced in association with a $Z$ boson [61, 62] and in vector boson fusion [63]. The current best bound reads $\mathrm{BR}(h \rightarrow$ invisible) $\lesssim 58 \% @ 95 \%$ C.L. [62] (see also [64] where a slightly stronger bound $\mathrm{BR}(h \rightarrow$ invisible $) \lesssim 40 \% @ 95 \%$ C.L. has been obtained, recasting a CMS stop search [65]). Bounds are expected to be improved down to $\mathrm{BR}(h \rightarrow$ invisible $) \lesssim 10 \%$ at the high luminosity LHC [66].

Due to the mixing with the Higgs boson, the dark scalar $s$ acquires in turn couplings to all SM particles that are suppressed by a factor $s_{\alpha}$ compared to the SM Higgs. Therefore, the dark scalar can be searched for at the LHC in the usual Higgs searches. Particularly strong constraints arise already from current searches in the $W W$ and $Z Z$ channels that exclude a signal strength of the order of $\mu \sim 0.1$ over a very broad range of masses [67-70]. The production cross section of the scalar is suppressed by $s_{\alpha}^{2}$ with respect to a SM Higgs boson with the same mass. Therefore, we generically expect a bound on the mixing angle of the order of $s_{\alpha} \lesssim 0.3$. This is slightly more stringent than the bound obtained from Higgs rate measurements, $c_{\alpha} \gtrsim 0.9$, discussed above.

Note, however, that also the dark scalar can decay into dark sector particles. The corresponding partial width into dark fermions and dark photons are given by the expressions in (5.2) and (5.3) with the replacements $m_{h} \rightarrow m_{s}$ and $s_{\alpha} \rightarrow c_{\alpha}$. If the scalar is light, with a mass below the $W W$ threshold, its decay width into SM particles is very small. Therefore, its invisible branching ratio can be sizable, in particular if the decay into dark fermions is kinematically accessible. This can easily reduce the branching ratio into SM particles by a factor of few or more and reduce the scalar signal strength well below $\mu_{s}=0.1$ also for mixing angles of $s_{\alpha} \gtrsim 0.3$. This is illustrated in the lower left plot of figure 3 that shows 

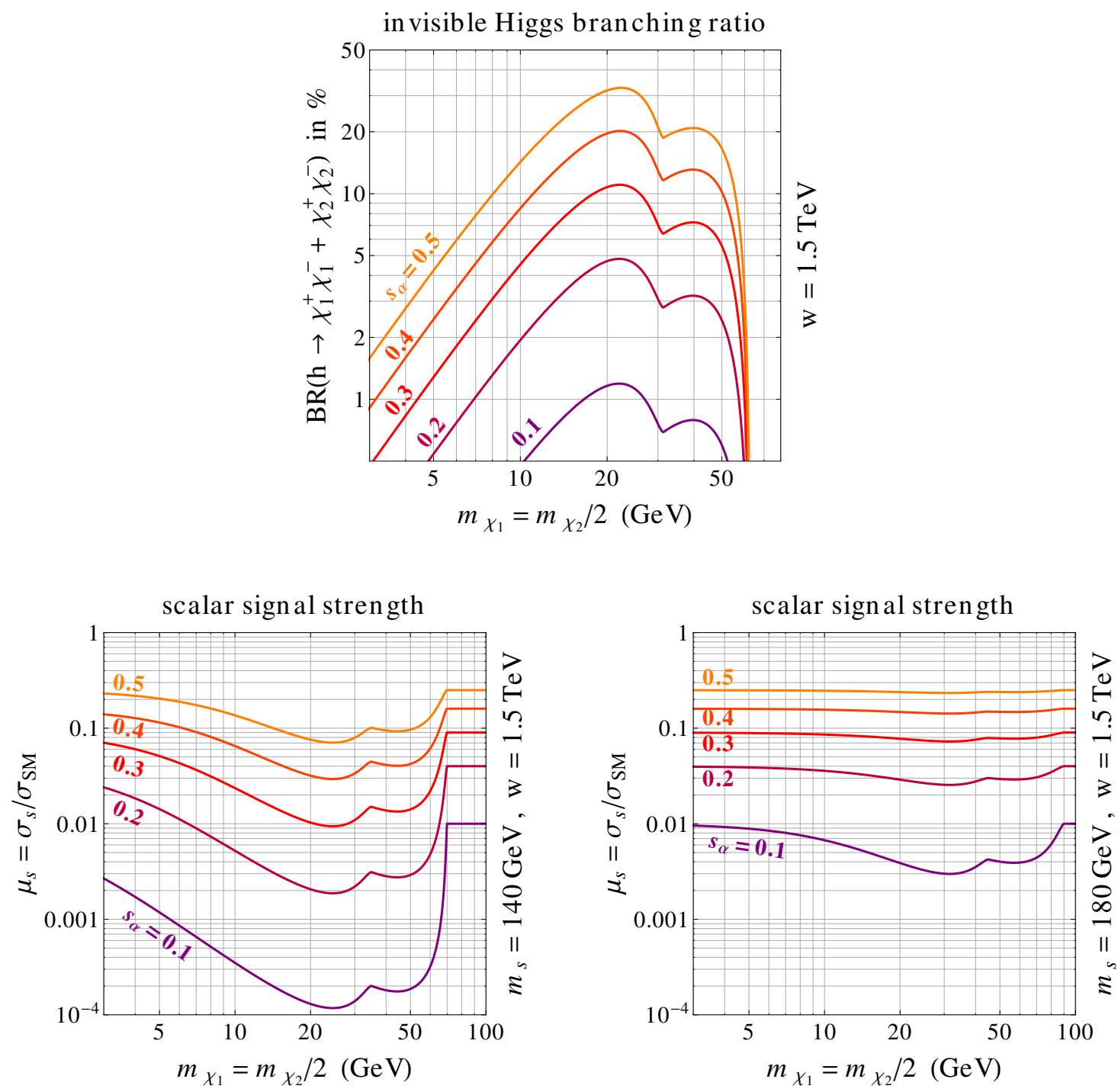

Figure 3. Top: the invisible branching ratio of the Higgs boson as a function of the charged dark fermion mass, for example choices of the scalar mixing angle. Bottom: the scalar signal strength into SM particles as function of the charged dark fermion mass for example choices of the scalar mixing angle. The scalar mass is fixed to $m_{s}=140 \mathrm{GeV}$ in the left and $m_{s}=180 \mathrm{GeV}$ in the right plot.

the signal strength of a $140 \mathrm{GeV}$ dark scalar for several choices of $s_{\alpha}$ as a function of the charged dark fermion masses $m_{\chi_{1}}=m_{\chi_{2}} / 2$. If the dark scalar has a mass above the $W W$ threshold, its width is dominated by decays into $W W$ and decays into dark fermions tend to give only a small correction. This is illustrated in the lower right plot of figure 3 , where we show the signal strength of the dark scalar for a dark scalar mass of $m_{s}=180 \mathrm{GeV}$.

In summary, we find that in the bulk of parameter space the prospects for detecting the dark scalar at the next run of the LHC are excellent, unless in the case where it dominantly decays into dark fermions. In the latter case, precision measurements of the Higgs signal strength in inclusive Higgs production will provide the strongest constraint on the mixing 
angle. In the case of sufficiently light dark fermions, a high luminosity LHC could provide sensitivity to the invisible decay of the Higgs boson.

\section{Dark matter and dark photon phenomenology}

The dark fermion sector of our model contains two charged and two neutral Dirac fermions $\chi_{1,2}$ and $\xi_{1,2}$. If the mass of each fermion is less than the sum of the other three masses, none of the fermions can decay and all four constitute a stable dark matter component. If one of the fermions has a mass that is larger than the sum of the other three masses, it can decay into the lighter three fermions through $W^{\prime}$ exchange. In that case the dark matter will consist of only the lighter three fermions. None of the other massive particles of the model are stable in the regions of parameter space that we will consider. The heavy dark gauge bosons can decay into a pair of dark fermions, while the dark scalar can decay through the Higgs portal into a pair of SM particles. The massless dark photon can have interesting effects in the early universe.

\subsection{Dark matter relic abundance}

The relic abundance of the charged dark fermions $\chi_{i}$ is primarily set by annihilation into two massless dark photons $\gamma^{\prime}$. Annihilation into two dark scalars is p-wave suppressed and typically negligible. Annihilation into SM particles through an s-channel exchange of the dark scalar or the Higgs is strongly suppressed by the small Higgs portal and therefore also negligible. ${ }^{6}$ For the annihilation cross section into dark photons, depicted in diagram (a) of figure 4 , we find

$$
(\sigma v)_{\chi_{i}} \simeq \frac{e_{X}^{4}}{8 \pi} \frac{1}{m_{\chi_{i}}^{2}}
$$

This annihilation cross section decreases for increasing charged dark fermion masses. The relic abundance of stable charged dark fermions is approximately given by

$$
\Omega_{\chi_{i}} h^{2} \simeq 0.12 \times\left(\frac{2.2 \times 10^{-26} \mathrm{~cm}^{3} / s}{(\sigma v)_{\chi_{i}}}\right)
$$

A charged dark fermion fraction of the total relic abundance is subject to various constraints [71-73]. A component of (strongly) self-interacting dark matter is constrained by halo shapes [74] and the observed structure of the Bullet Cluster [75, 76]. Numerical simulations that account for the observed deviations from spherical halos allow for $\sim 10 \%$ interacting dark matter, while simulations of the Bullet Cluster allow for up to $\sim 30 \%$ of all dark matter to have arbitrarily strong self-interactions. A more stringent bound comes from possible CMB structure, which constrains the fraction of dark matter coupled to dark radiation to $\lesssim 5 \%$ [77]. If dark matter forms a disk due to long ranged interactions, the local dark matter density puts a comparable bound on this fraction [71]. In the following,

\footnotetext{
${ }^{6}$ Dark matter annihilation into SM particles through s-channel exchange of the Higgs or the dark scalar might be important in fine tuned corners of parameter space where the annihilation is resonant, e.g. $m_{\chi_{i}} \simeq$ $m_{h} / 2$ or $m_{\chi_{i}} \simeq m_{s} / 2$.
} 


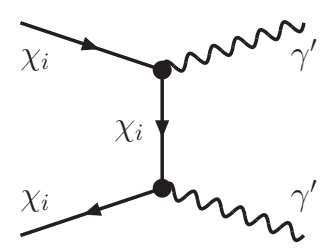

(a)

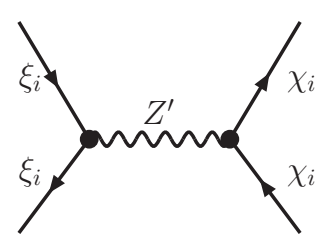

(b)

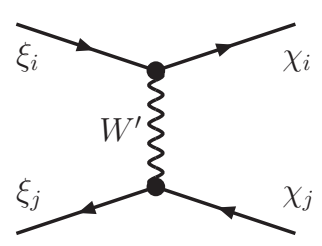

(c)

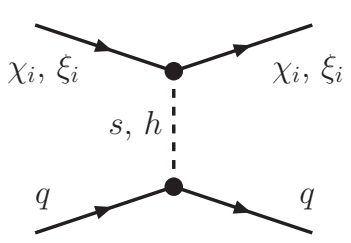

$(d)$

Figure 4. Feynman diagrams corresponding to the dominant processes contributing to dark matter annihilation (a), (b), and (c), as well as direct detection (d). In the case of annihilation into dark photons (a) an additional crossed diagram is not shown.

we will therefore allow a charged dark matter fraction of at most $5 \%$. This leads to an upper bound on the mass of the charged dark fermions. We find

$$
m_{\chi_{1}}^{2}+m_{\chi_{2}}^{2} \lesssim(1 \mathrm{TeV})^{2} \times e_{X}^{4}
$$

For values of the dark electromagnetic coupling of the order of the electroweak couplings of the SM, $e_{X} \sim 0.5$, this implies an upper bound on the mass of stable charged dark fermions of a few $100 \mathrm{GeV}$.

It is important to observe, that in the absence of the dark photons, the annihilation cross section of the fermions $\chi_{i}$ would be strongly suppressed resulting generically in a dark matter relic abundance in excess of the measured value $\Omega_{\mathrm{DM}} h^{2} \simeq 0.12$.

Obviously, the neutral dark fermions $\xi_{i}$ cannot annihilate into the dark photons at tree level. Annihilation into two dark scalars or into SM particles is also suppressed for the same reasons as in the case of the charged dark fermions. The only unsuppressed annihilation of the neutral dark fermions is into the charged dark fermions, which is only an option if the neutral fermions are significantly heavier than the charged ones, such that their freeze out occurs sufficiently earlier. Annihilation into charged dark fermions can proceed through s-channel exchange of a $Z^{\prime}$ or t-channel exchange of a $W^{\prime}$ as shown in diagrams (b) and (c) in figure 4. The s-channel exchange of a dark scalar is suppressed by the charged fermion Yukawa coupling and hardly relevant in the regions of parameter space that we will consider. Even more suppressed is the s-channel annihilation through a Higgs boson. In the limit $m_{\chi_{i}} \ll m_{\xi_{i}} \ll m_{Z^{\prime}}, m_{W^{\prime}}$, the annihilation cross section is approximately given by

$$
(\sigma v)_{\xi_{i}} \simeq \frac{m_{\xi_{i}}^{2}}{2 \pi w^{4}}\left(4 s_{X}^{4}-3 s_{X}^{2}+2\right) .
$$

We learn that the annihilation cross section increases for increasing neutral dark fermion mass. For $m_{\xi_{i}} \sim m_{Z^{\prime}} / 2$, the annihilation cross section is strongly enhanced by the $Z^{\prime}$ resonance and reaches its maximum. For $m_{\xi_{i}} \gtrsim m_{Z^{\prime}} / 2$ the annihilation cross section decreases again with increasing mass. Expressions for the annihilation cross section that hold in the general case of arbitrary fermion and gauge boson masses are given in the appendix D.

The relic abundance of the stable neutral dark fermions is given by an expression analogous to (6.2). The relic abundance of the light neutral dark matter species is shown 

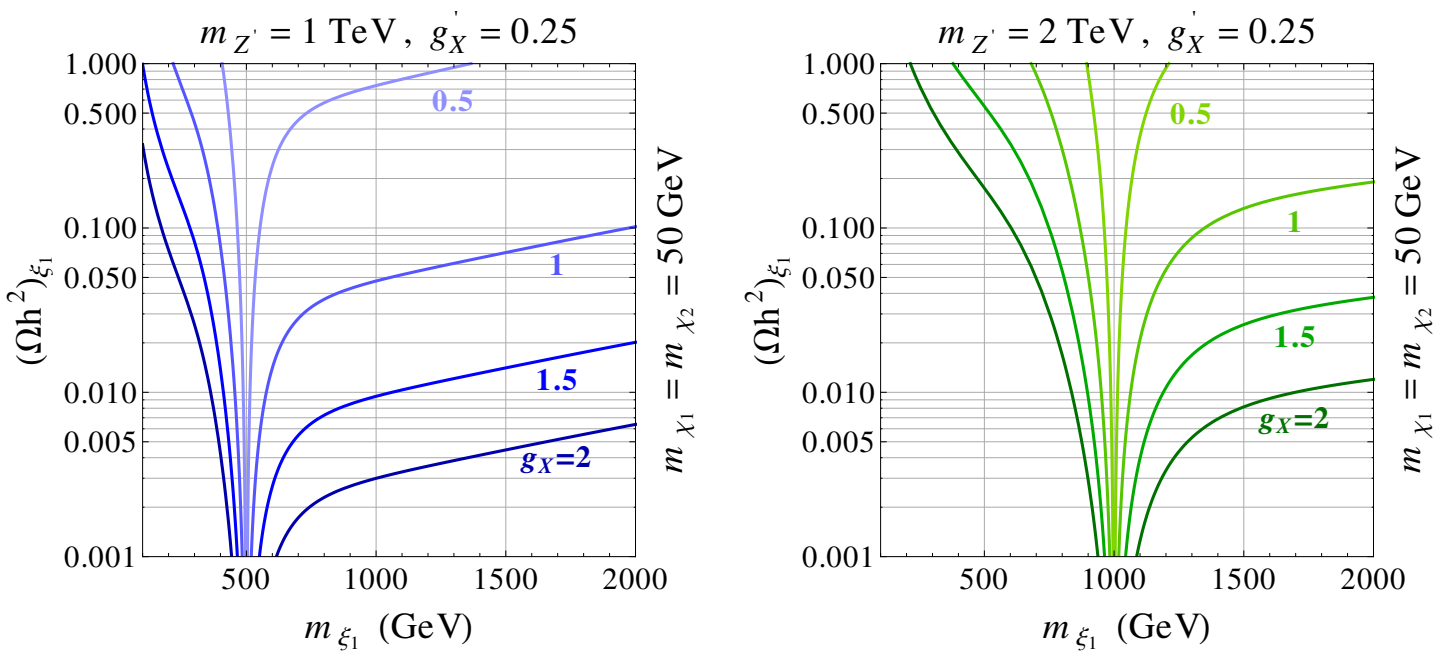

Figure 5. The relic density of the light neutral dark fermion species as a function of its mass. In the left (right) plot, the $Z^{\prime}$ mass is fixed to $m_{Z^{\prime}}=1(2) \mathrm{TeV}$.

in the plots of figure 5 as a function of the fermion mass. In the left and right plots the $Z^{\prime}$ mass is fixed to $m_{Z^{\prime}}=1 \mathrm{TeV}$ and $m_{Z^{\prime}}=2 \mathrm{TeV}$, respectively. The charged fermion masses are fixed to $m_{\chi_{1}}=m_{\chi_{2}}=50 \mathrm{GeV}$ and the dark hypercharge gauge coupling is $g_{X}^{\prime}=0.25$. The various curves correspond to different choices of the dark $\mathrm{SU}(2)_{X}$ gauge coupling that ranges from $g_{X}=0.5$ up to $g_{X}=2$. We observe that the annihilation into charged fermions is very efficient. If the neutral fermion is above the $Z^{\prime}$ resonance, the requirement of the right relic abundance leads to an upper bound on the $\mathrm{SU}(2)_{X}$ gauge coupling of the order of $g_{X} \lesssim 1$. Note that a dark matter fermion with mass above the $Z^{\prime}$ mass implies a large fermion Yukawa coupling and therefore generically leads to a UV instability in the dark scalar quartic $\lambda_{\Sigma}$. For a dark fermion mass below the $Z^{\prime}$ resonance as preferred by vacuum stability, gauge couplings over a broad range of values can be made easily compatible with the relic abundance.

\subsection{Dark matter direct detection}

The dark matter particles couple to SM particles only through the Higgs portal. The direct detection cross section is therefore necessarily suppressed by the mixing between the Higgs and the dark scalar. Working with scalar mass eigenstates and evaluating the relevant diagram in figure 4, we find for the spin-independent cross section for elastic scattering of neutral dark matter particles $\xi_{i}$ off protons

$$
\sigma_{\mathrm{SI}}=\frac{Y_{\xi_{i}}^{2}}{2 \pi} \frac{m_{\xi_{i}}^{2} m_{p}^{4}}{v^{2}\left(m_{\xi_{i}}+m_{p}\right)^{2}} f^{2} s_{\alpha}^{2} c_{\alpha}^{2}\left(\frac{1}{m_{h}^{2}}-\frac{1}{m_{s}^{2}}\right)^{2},
$$

where $m_{p}$ is the proton mass and

$$
f=\frac{2}{9}+\frac{7}{9}\left(f_{T_{u}}+f_{T_{d}}+f_{T_{s}}\right) \simeq 0.3
$$

parametrizes the nuclear matrix element $[78,79]$. The dark matter direct detection cross section is suppressed by the scalar mixing angle $s_{\alpha}^{2}$ as well as by the destructive interference 
between the Higgs and dark scalar exchange. We find typical direct detection signals at the level of $\sigma_{\mathrm{SI}} \simeq 10^{-46}-10^{-47} \mathrm{~cm}^{2}$, well below the current experimental sensitivities of the XENON100 experiment [80] and the LUX experiment [81]. The predicted signals are probably also below the sensitivity of XENON1T [82]. They should however be in reach of the planned LZ experiment [83].

An equation completely analogous to (6.5) holds also for the direct detection cross section of the charged dark matter fermions $\chi_{i}$. However, barring additional structure which radically changes the local density of the charged dark matter component [71], the maximal relic density fraction of $5 \%$ strongly suppresses sensitivity of direct detection experiments to the $\chi_{i}$. For charged dark matter masses of $m_{\chi_{i}} \sim 50 \mathrm{GeV}$, there are only corners of parameter space, where the direct detection cross section of the charged fermions might reach $\sigma_{\mathrm{SI}} \sim$ few $\times 10^{-48} \mathrm{~cm}^{2}$. Combined with the smaller density of charged dark matter, this results in direct detection rates that are at the border of or even below the atmospheric and supernova neutrino background, and beyond the reach of planned direct detection experiments.

\subsection{Number of relativistic degrees of freedom in the early universe}

The dark photon of our model contributes to the effective number of relativistic degrees of freedom in the early universe. Measurements of the ${ }^{4} \mathrm{He}$ abundance [84] in the universe and the combination of Planck data with astrophysical measurements of the Hubble constant [85] put constraints on the active degrees of freedom during Big Bang nucleosynthesis $(\mathrm{BBN})$ and at the time at which the Cosmic Microwave Background (CMB) radiation formed, respectively $[71,86]$.

The process of scattering of visible photons into dark photons $\gamma \gamma \leftrightarrow \gamma^{\prime} \gamma^{\prime}$ can potentially keep the dark and the visible sector in thermal equilibrium. In our model, the Higgs portal is the only connection between the dark and the visible sectors. Therefore $\gamma \gamma \leftrightarrow \gamma^{\prime} \gamma^{\prime}$ is induced by a dimension eight operator and suppressed by two loops. As a consequence, this process decouples at very high temperatures. More relevant processes that connect the dark and the visible sector are the annihilation of visible photons into dark fermions, $\gamma \gamma \leftrightarrow \bar{\chi} \chi$, and of dark photons into SM fermions, $\gamma^{\prime} \gamma^{\prime} \leftrightarrow \bar{f} f$. Such processes are induced by dimension six operators and only suppressed by one loop. Depending on the dark scalar mass, mixing angle and the dark fermion Yukawa couplings, we find that the decoupling temperature is at the order of $T\left(t_{\mathrm{dec}}\right) \sim \mathcal{O}(10) \mathrm{GeV}$. Below this temperature, the entropy density should be separately conserved in both sectors, so that the ratio of temperatures $\xi(t)=T_{\text {dark }} / T_{\text {vis }}$ in the dark and visible sector at some later time $t$ is given by [71]

$$
\xi(t)=\left(\frac{g_{* s}^{\mathrm{dark}}\left(t_{\mathrm{dec}}\right)}{g_{* s}^{\mathrm{vis}}\left(t_{\mathrm{dec}}\right)} \frac{g_{* s}^{\mathrm{vis}}(t)}{g_{* s}^{\mathrm{dark}}(t)}\right)^{1 / 3}
$$

where $g_{* s}(t)$ denotes the effective number of degrees of freedom at the time $t$. In the SM, all degrees of freedom besides the Higgs boson, the top, and the electroweak gauge bosons are active during decoupling, so that $g_{* s}^{\mathrm{vis}}\left(t_{\mathrm{dec}}\right)=86.75$. In the dark sector, the dark photons, and dark fermions can contribute $g_{* s}^{\text {dark }}\left(t_{\text {dec }}\right)=2+\frac{7}{8} \times 4 \times n=2+n \times 3.5$, where $n$ is the number of dark fermions with masses below the decoupling temperature. At the BBN 
scale, electrons, neutrinos and photons contribute to the SM entropy density $g_{* s}^{\mathrm{vis}}\left(t_{\mathrm{BBN}}\right)=$ $\frac{7}{8} \times 10+2=10.75$, while during formation of the CMB only colder neutrinos and photons remain active $g_{* s}^{\text {vis }}\left(t_{\mathrm{CMB}}\right)=\left(\frac{4}{11}\right)^{4 / 3} \times \frac{7}{8} \times 6+2=3.36$. In the dark sector, at these times only the dark photon is a relativistic degree of freedom, $g_{* s}^{\text {dark }}\left(t_{\mathrm{BBN}}\right)=g_{* s}^{\text {dark }}\left(t_{\mathrm{CMB}}\right)=2$. The temperatures in the dark sector during BBN and CMB are therefore smaller than in the visible sector. We find

$$
\xi\left(t_{\mathrm{BBN}}\right) \approx 0.50 / 0.70 / 0.82, \quad \xi\left(t_{\mathrm{CMB}}\right) \approx 0.34 / 0.47 / 0.56
$$

where the first/second/third value corresponds to $n=0 / 1 / 2$. These temperature ratios can be translated into the change of effective number of neutrinos at these temperatures

$$
\begin{aligned}
\Delta N_{\mathrm{eff}, \nu}^{\mathrm{BBN}} & =\frac{8}{7} \xi\left(t_{\mathrm{BBN}}\right)^{4} \approx 0.07 / 0.27 / 0.53, \\
\Delta N_{\mathrm{eff}, \nu}^{\mathrm{CMB}} & =\left(\frac{4}{11}\right)^{-\frac{4}{3}} \frac{8}{7} \xi\left(t_{\mathrm{CMB}}\right)^{4} \approx 0.06 / 0.22 / 0.43 .
\end{aligned}
$$

In the Standard Model, the effective number of neutrinos is given by $N_{\text {eff, } \nu}=N_{\text {eff, } \nu}^{\mathrm{BBN}}=$ $N_{\mathrm{eff}, \nu}^{\mathrm{CMB}}=3.046$ [87]. Currently, the strongest constraints on the numbers of effective degrees of freedom during BBN [84] and CMB [85] are

$$
\begin{aligned}
& N_{\mathrm{eff}, \nu}^{\mathrm{BBN}}=3.24_{-0.57}^{+0.61} \text { at } \quad 68 \% \text { C.L. }, \\
& N_{\mathrm{eff}, \nu}^{\mathrm{CMB}}=3.52_{-0.45}^{+0.48} \text { at } 95 \% \text { C.L. . }
\end{aligned}
$$

This has to be compared with the values for $N_{\mathrm{eff}, \nu}+\Delta N_{\mathrm{eff}, \nu}$ in our model, which can be comfortably accommodated within the uncertainties. Future CMB experiments will improve the bounds on $N_{\mathrm{eff}, \nu}^{\mathrm{CMB}}$ significantly [88] and might be able to find evidence for the presence of the dark photon in the early universe.

\section{Numerical analysis, discussion, and outlook}

We now analyse the dark scalar and dark matter phenomenology of the model numerically, starting from the underlying model parameters in the Lagrangian. We explore regions of parameter space that are compatible with vanishing Higgs and scalar quartic couplings at the Planck scale. We checked that small non-zero scalar quartics at the Planck scale do not appreciably change any of our findings. In addition, we impose the correct dark matter relic abundance with a $\sim 5 \%$ admixture of dark charged dark matter component.

The model introduced in section 4 has 9 free parameters: the Higgs quartic $\lambda_{H}$, the dark scalar quartic $\lambda_{\Sigma}$, the portal coupling $\lambda_{H \Sigma}$, the $\mathrm{SU}(2)_{X} \times \mathrm{U}(1)_{X}$ gauge couplings $g_{X}$ and $g_{X}^{\prime}$, as well as the Yukawa couplings of the dark fermions $Y_{\chi_{1}}, Y_{\chi_{2}}, Y_{\xi_{1}}$, and $Y_{\xi_{2}}$. We consider the one loop effective scalar potential given in the appendix A, with the approximations discussed there. We use 2 loop beta functions for the SM couplings and 1 loop beta functions for the dark sector couplings. For every given set of parameters, we minimize the effective potential numerically and obtain values for the vevs $v$ and $w$, as well as mass eigenvalues for the scalars and their mixing angle. 
We allow to vary the Higgs quartic coupling at the electroweak scale in the range $0.259 \lesssim \lambda_{H}\left(m_{t}\right) \lesssim 0.288$ which is compatible with a vanishing $\lambda_{H}$ at the Planck scale given the current uncertainties on the top mass and the strong gauge coupling. The portal coupling $\lambda_{\Sigma H}$ sets the ratio of the Higgs vev $v$ and the dark vev $w$. For a dark vev at the TeV scale, the portal coupling is small, typically at the order of $\left|\lambda_{\Sigma H}\right| \sim 10^{-2}$. We chose a vanishing dark scalar quartic coupling $\lambda_{\Sigma}$ at the Planck scale. The value of the scalar quartic coupling at the weak scale as well as the value of its beta function are mainly determined by the $\mathrm{SU}(2)_{X}$ gauge coupling $g_{X}$ and the largest dark fermion Yukawa coupling $Y_{\xi_{2}}$. We chose these parameters such that we reproduce the Higgs vev of $v=246 \mathrm{GeV}$ as well as a Higgs mass of $124.5 \mathrm{GeV} \lesssim m_{h} \lesssim 126.5 \mathrm{GeV}$. With these boundary conditions the heaviest neutral dark fermion turns out to be unstable. The lighter neutral dark fermion comprises the dominant part of the dark matter relic abundance. Obtaining the right annihilation cross section fixes its Yukawa coupling $Y_{\xi_{1}}$. We allow to vary $Y_{\xi_{1}}$ such that its annihilation cross section lies in the range $1.8 \times 10^{-26} \mathrm{~cm}^{3} / \mathrm{s}<(\sigma v)_{\xi_{1}}<2.6 \times 10^{-26} \mathrm{~cm}^{3} / \mathrm{s}$, reproducing the right dark matter abundance within approximately $20 \%$. For simplicity we assume degeneracy among the charged dark matter particles and fix their Yukawa couplings $Y_{\chi_{1}}, Y_{\chi_{2}}$ such that their masses are $m_{\chi_{1}}=m_{\chi_{2}}=50 \mathrm{GeV}$. With this mass, requiring that the charged dark matter is responsible for $\sim 5 \%$ of the observed relic abundance fixes the $\mathrm{U}(1)_{X}$ gauge coupling at the TeV scale to be at the order of $g_{X}^{\prime} \sim 0.25$. Such small values of $g_{X}^{\prime}$ do not significantly impact the running of the dark scalar quartic, and therefore cannot overcome the effect of the largest dark fermion Yukawa coupling $Y_{\xi_{2}}$. As a consequence the dark scalar quartic will cross zero at the Planck scale. The limiting case where the scalar quartic coupling barely touches zero close to the Planck scale demands a coupling of $g_{X}^{\prime} \sim 0.7$, which results in a charged dark matter component well below the percent level. We find that the low energy phenomenology of the dark scalar $s$ and the dominant dark matter component $\xi_{1}$ hardly depends on these choices.

Under the discussed boundary conditions, we obtain predictions for the mass and signal strength of the dark scalar, as well as the mass and direct detection cross section of the dominant dark matter component. In figure 6, we show the predictions for direct searches for the dark scalar at the LHC (left) as well as dark matter direct detection experiments (right). The red/green/blue points correspond to different choices for the dark scalar vev $w=1 / 1.5 / 2 \mathrm{TeV}$ as indicated. For a fixed choice of $w$, all parameters of the model are determined by the chosen boundary conditions discussed above. Demanding that central values for $\lambda_{H}\left(m_{t}\right), m_{h}$, and, $(\sigma v)_{\xi_{1}}$ are reproduced, we obtain the predictions indicated by the stars in the plots of figure 6 . The dark and light points show the ranges of predictions that can be obtained by varying the Higgs quartic between $0.266<\lambda_{H}\left(m_{t}\right)<0.280$ and $0.259<\lambda_{H}\left(m_{t}\right)<0.288$, which corresponds to the $1 \sigma$ and $2 \sigma$ ranges for $m_{t}$ and $\alpha_{s}$.

We find dark scalar masses in the range $140 \mathrm{GeV} \lesssim m_{s} \lesssim 220 \mathrm{GeV}$. The current sensitivity of Higgs searches in the $h \rightarrow Z Z$ and $h \rightarrow W W$ channels at the LHC [67-70] already starts to probe parts of parameter space. Improving the sensitivity down to the percent level over the shown mass range, would probe almost the entire parameter space of the discussed scenario. Note that in this scenario, the charged dark matter mass is fixed to $50 \mathrm{GeV}$, allowing for a sizable $s \rightarrow \chi_{i} \chi_{i}$ rate that dilutes the signal strength at the 

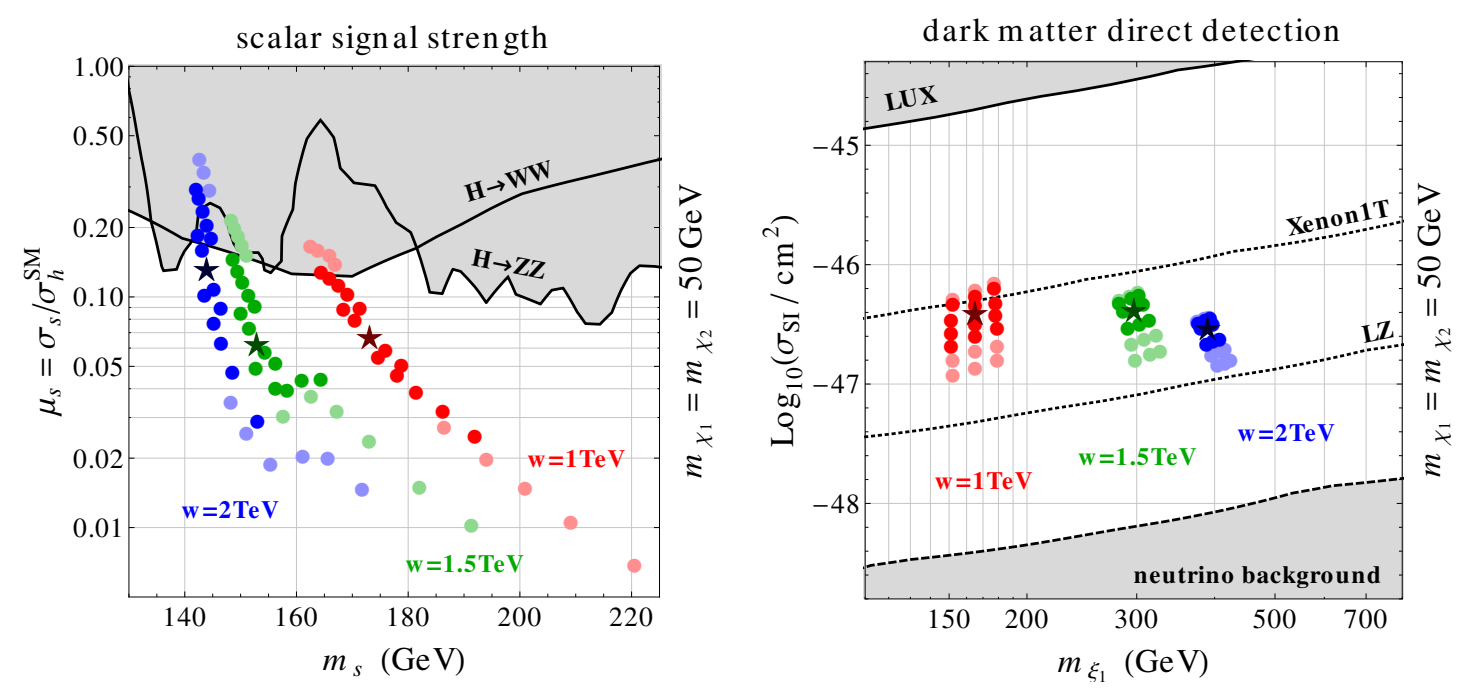

Figure 6. Left: predictions for the signal strength of the dark scalar as function of its mass. The shaded regions are excluded by Higgs searches at the LHC [67-70]. Right: predictions for the spin independent dark matter nucleon cross section. The region above the solid black line is excluded by the current experimental bound from LUX [81]. Future sensitivities of XENON1T [82] and LZ [83] are indicated with the dotted lines. In the region below the dashed line, neutrino background limits the sensitivity of direct detection experiments.

LHC. For charged dark matter particles that are heavier than half the dark scalar mass, the dark scalar signal strength increases, especially for dark scalar masses below the $W W$ threshold, $m_{s} \lesssim 160 \mathrm{GeV}$. As expected from the discussion in section 5 , the prospects for direct detection of the dark scalar at the next run of the LHC are excellent.

Typical values for the dark matter mass are at the level of few $100 \mathrm{GeV}$. As anticipated already in section 6.2, the predicted direct detection cross sections are still 1-2 orders of magnitude below the current best experimental sensitivity of the LUX experiment [81]. The XENON1T experiment [82] might start to probe parts of the parameter space, if the dark scalar vev is around $w \simeq 1 \mathrm{TeV}$ or smaller, which corresponds to a small dark matter mass of around $m_{\xi_{1}} \simeq 150 \mathrm{GeV}$. We expect that future dark matter direct detection experiments like LZ [83] will be able to detect the dark matter unless the dark vev is far above the $\mathrm{TeV}$ scale, in which case the direct searches for the dark scalar become more powerful. The direct detection rates of the charged dark matter component is generically below the neutrino floor. Interestingly, for $m_{\chi} \leq m_{h} / 2$ the Higgs can decay into the light dark matter candidate with a sizable branching fraction. Improved measurements of the invisible branching ratio of the Higgs can therefore indirectly constrain the mass and fraction of charged dark matter even if direct detection experiments cannot see it.

Anomaly cancellation enforces at least two generations of charged fermions with dark charges $Q_{X}= \pm 1$. In the discussed model they are both stable. As argued in [71], this can result in dark bound states, which imply cosmological dynamics radically different from cold dark matter. This would provide a new testing ground for our model through measurements of the dark matter distribution within the milky way, for example by precisely mapping the 
movement of stars as planned by the GAIA survey [89]. Numerical simulations of galaxy formation are beyond the scope of this work, but we observe that a double disk scenario as discussed in [71] can be reproduced within the parameter space of our model.

If all physics below the Planck scale should be captured in a model without new mass scales, the matter-antimatter asymmetry needs to be addressed. One possibility would be to consider baryogenesis at the electroweak scale. This requires a strongly first order phase transition [90, 91]. Since the electroweak scale is induced by a dynamically broken dark gauge symmetry, we expect bubble nucleation to occur through a two step process in the discussed model. Similar scenarios have been studied in general singlet extensions of the SM [92, 93] and in a model with an additional electroweak scalar triplet [94]. The sphaleron rate for the dark gauge group differs from the one in the SM. A direct comparison to previous results is therefore not straightforward and we leave this interesting question for future work.

\section{Conclusion}

After the $8 \mathrm{TeV}$ run of the LHC, the dynamics of the electroweak symmetry breaking mechanism is still a mystery. Natural UV completions of the Standard Model predict new degrees of freedom in the vicinity of the electroweak scale, that have not been discovered so far. If the electroweak scale emerges as a quantum effect from a boundary condition of vanishing mass parameters at the Planck scale, the large disparity of scales can be explained by RGE running similar to the case of the QCD scale. Unlike QCD however, there is no underlying symmetry which protects such a boundary condition at the Planck scale in the SM.

We have shown that clasically scale invariant models with a minimal dark sector, that incorporate radiative electroweak symmetry breaking through a Higgs portal, and stabilize the Higgs potential up to the Planck scale put an upper bound of the order of a few $\mathrm{TeV}$ on the mass of the dark matter candidate. As discussed previously in the literature, if the dark sector consists of only a scalar charged under dark gauge interactions, the dark gauge bosons that obtain their mass through couplings to the scalar can constitute dark matter. As the dark gauge coupling has to be sizable in order to generate Coleman-Weinberg symmetry breaking, in those scenarios one finds a lower bound on the dark matter mass. As a consequence, dark matter is generically constrained to a window of a few hundred $\mathrm{GeV}$ to a few $\mathrm{TeV}$.

In this work we have considered the effect of additional fermions in the dark sector. Radiative symmetry breaking dictates that the bosonic contribution to the effective potential needs to be larger than the fermionic contribution. This renders the fermions naturally lighter than the gauge bosons and allows for fermionic dark matter masses at the electroweak scale, or even below. We demonstrated this on the basis of a model that contains a dark sector with a $\mathrm{SU}(2) \times \mathrm{U}(1)$ gauge group, a dark scalar that is a doublet under the dark SU(2), and two generations of chiral dark fermions. The gauge interactions drive the dark scalar quartic negative at low energies and radiatively induce a vev for the dark scalar. The dark sector gauge symmetry is broken spontaneously by the vev of the scalar doublet, 
$\mathrm{SU}(2) \times \mathrm{U}(1) \rightarrow \mathrm{U}(1)$, leaving a long-ranged "dark electromagnetism" at low energies. The vev of the scalar doublet also generates a Higgs mass term through a quartic portal coupling and thus triggers breaking of the electroweak symmetry in the visible sector.

If this is indeed the origin of electroweak symmetry breaking, the dark scalar mixes with the Higgs and therefore has Higgs-like couplings to Standard Model particles, only suppressed by the portal coupling. We find that if the dark scalar stabilizes the vacuum up to the Planck scale, its mass is constrained to be $m_{s} \lesssim 250 \mathrm{GeV}$. Its signal strength is generically at the level of $\mathcal{O}(10 \%)$ of a SM Higgs boson. Current Higgs searches in the $W W$ and $Z Z$ channels already constrain parts of the parameter space of the model, and the prospects for detecting the dark scalar at the next run of the LHC are excellent. Mixing of the dark scalar with the Higgs also leads to a slight reduction of the signal strenghts of the Higgs boson and more precise measurements of the various Higgs signal strengths are equally important to test the discussed framework. For sizable mixing, the Higgs boson can also decay through the scalar portal into the dark charged fermions, if they are kinematically accessible. This can induce an invisible branching ratio of the Higgs of up to $\sim 10 \%$ which can be within reach of the high-luminosity LHC.

The model has two dark matter components: (i) dark fermions that are charged under the long range dark electromagnetism and with masses typically below the electroweak scale, $m_{\chi_{i}} \lesssim 100 \mathrm{GeV}$; (ii) a neutral dark fermion with mass generically in the range $m_{\xi_{1}} \sim 100-500 \mathrm{GeV}$. The model can easily accommodate a non-negligible fraction of longrange interacting dark matter of the order of a few percent and could have interesting implications for galaxy structure formations. While the neutral dark matter component has a spin-independent scattering cross section with nuclei in reach of future direct detection experiments like XENON1T or LZ, the light dark matter component will be most likely buried in the neutrino background. In addition, the dark radiation present in the model can be independently tested by future measurements of the number of relativistic degrees of freedom in the early universe. Interestingly, the parameter space of our model in which direct detection experiments are least sensitive is the one most strongly constrained by collider searches for the dark scalar. The complementarity of both searches imply excellent prospects to discover or exclude our model in the near future.

Finally, we argue, that if the electroweak scale is generated subsequently to the breaking of a dark gauge symmetry, bubble nucleation during the dark and electroweak phase transition becomes a two step process. Previous studies of a similar scenario suggest, that a strong first order phase transition as required by electroweak baryogenesis can be achieved in this setup. Studies in this direction are left for future work.

\section{Acknowledgments}

We acknowledge helpful discussions with Prateek Agrawal, Gia Dvali, Steve Giddings, Chris Hill, Pedro Schwaller, Jessie Shelton and Alessandro Strumia. WA, MB and JL acknowledge the hospitality and support of the Theoretical Physics Group at SLAC. MC acknowledges the hospitality of MITP. WA, MC and JL acknowledge the hospitality of the Aspen Center for Physics and partial support by the National Science Foundation Grant 
No. PHYS-1066293. Fermilab is operated by Fermi Research Alliance, LLC, under contract DE-AC02-07CH11359 with the United States Department of Energy. The research of WA was supported by the John Templeton Foundation. Research at Perimeter Institute is supported by the Government of Canada through Industry Canada and by the Province of Ontario through the Ministry of Economic Development \& Innovation. MB acknowledges the support of the Alexander von Humboldt Foundation.

\section{A Effective potential}

The one loop effective potential $V_{\text {eff }}$ of our model is approximately given by

$$
\begin{aligned}
& V_{\text {eff }}(h, s) \simeq \frac{1}{8} \lambda_{H}\left(\mu_{h}\right) h^{4}+\frac{1}{4} \lambda_{\Sigma H}\left(\mu_{s h}\right) h^{2} s^{2}+\frac{1}{8} \lambda_{\Sigma}\left(\mu_{s}\right) s^{4} \\
& +\frac{1}{16 \pi^{2}}\left\{-3 m_{t}^{2}\left[\log \left(\frac{m_{t}^{2}}{\mu_{h}^{2}}\right)-\frac{3}{2}\right]\right. \\
& \left.+\frac{3}{2} m_{W}^{2}\left[\log \left(\frac{m_{W}^{2}}{\mu_{h}^{2}}\right)-\frac{5}{6}\right]+\frac{3}{4} m_{Z}^{2}\left[\log \left(\frac{m_{Z}^{2}}{\mu_{h}^{2}}\right)-\frac{5}{6}\right]\right\} \\
& +\frac{1}{16 \pi^{2}}\left\{-\sum_{i} m_{\chi_{i}}^{2}\left[\log \left(\frac{m_{\chi_{i}}^{2}}{\mu_{s}^{2}}\right)-\frac{3}{2}\right]-\sum_{i} m_{\xi_{i}}^{2}\left[\log \left(\frac{m_{\xi_{i}}^{2}}{\mu_{s}^{2}}\right)-\frac{3}{2}\right]\right. \\
& \left.+\frac{3}{2} m_{W^{\prime}}^{2}\left[\log \left(\frac{m_{W^{\prime}}^{2}}{\mu_{h}^{2}}\right)-\frac{5}{6}\right]+\frac{3}{4} m_{Z^{\prime}}^{2}\left[\log \left(\frac{m_{Z^{\prime}}^{2}}{\mu_{h}^{2}}\right)-\frac{5}{6}\right]\right\},
\end{aligned}
$$

where the field dependent masses are given by

$$
\begin{aligned}
m_{t}^{2} & =Y_{t}^{2} h^{2} / 2, \quad m_{W}^{2}=g^{2} h^{2} / 4, \quad m_{Z}^{2}=\left(g^{2}+\left(g^{\prime}\right)^{2}\right) h^{2} / 4, \\
m_{\chi_{i}}^{2}=Y_{\chi_{i}}^{2} s^{2} / 2, \quad m_{\xi_{i}}^{2} & =Y_{\xi_{i}}^{2} s^{2} / 2, \quad m_{W^{\prime}}^{2}=g_{X}^{2} s^{2} / 4, \quad m_{Z^{\prime}}^{2}=\left(g_{X}^{2}+\left(g_{X}^{\prime}\right)^{2}\right) s^{2} / 4 .
\end{aligned}
$$

In (A.1) we took into account contributions from the top quark, the $W$ and $Z$ bosons, the dark fermions and the dark $W^{\prime}$ and $Z^{\prime}$ bosons. Contributions to the effective potential from the Higgs boson $h$, the scalar $s$, and the corresponding Goldstone bosons lead to imaginary parts of the one loop effective potential, whenever the corresponding quartic coupling $\left(\lambda_{H}\right.$ or $\lambda_{\Sigma}$ ) becomes negative. Such imaginary parts signal the presence of an instability in the potential [95]. ${ }^{7}$ We neglect the contributions from $h, s$ and the corresponding Goldstone bosons. We explicitly checked that this leads to shifts in physical observables of a few percent at most. We also do not take into account additional corrections coming from the anomalous dimensions of the Higgs and the scalar field, as they are typically only at the few percent level, as well.

All couplings as well as all logarithms in the effective potential depend on a renormalization scale. In (A.1) we introduced three scales $\mu_{h}, \mu_{s}$, and $\mu_{h s}$. In the absence of the portal coupling $\lambda_{\Sigma H}$ it is clear that the dependencies on the renormalization scales $\mu_{h}$ and $\mu_{s}$ cancel separately up to terms suppressed by two loops. In our numerical analysis

\footnotetext{
${ }^{7}$ The imaginary part coming from the Goldstone contribution in the SM is actually spurious and can be avoided by resummation [96, 97].
} 
we set these renormalization scales to the corresponding field values $\mu_{h}=h$ and $\mu_{s}=s$, which is expected to keep higher order corrections to the effective potential small. Switching on the portal coupling will lead to residual scale dependencies at the one loop level. However, such effects are strongly suppressed due to the smallness of the portal coupling and they are of the same order as the neglected effects from scalar loops on the effective potential. The scale dependence of the portal coupling itself is very weak and in the numerical analysis we chose $\mu_{h s}=\sqrt{h s}$. Refined methods for analyzing effective potentials were recently presented in [98]. We do not expect them to change any of our conclusions in a significant way.

\section{B Beta functions}

The one loop beta functions of the couplings of our framework read $(t=\log \mu)$

$$
\begin{aligned}
\frac{d \lambda_{H}}{d t}=\beta_{\lambda_{H}}=\beta_{\lambda_{H}}^{\mathrm{SM}}+ & \frac{1}{16 \pi^{2}} 4 \lambda_{\Sigma H}^{2}, \\
\frac{d \lambda_{\Sigma}}{d t}=\beta_{\lambda_{\Sigma}}= & \frac{1}{16 \pi^{2}}\left(12 \lambda_{\Sigma}^{2}+4 \lambda_{\Sigma H}^{2}-9 g_{X}^{2} \lambda_{\Sigma}-3\left(g_{X}^{\prime}\right)^{2} \lambda_{\Sigma}+\frac{9}{4} g_{X}^{4}+\frac{3}{4}\left(g_{X}^{\prime}\right)^{4}+\frac{3}{2} g_{X}^{2}\left(g_{X}^{\prime}\right)^{2}\right. \\
& \left.\quad-4 \sum_{i}\left(Y_{\xi_{i}}^{4}+Y_{\chi_{i}}^{4}\right)+4 \lambda_{\Sigma} \sum_{i}\left(Y_{\xi_{i}}^{2}+Y_{\chi_{i}}^{2}\right)\right), \\
\frac{d \lambda_{\Sigma H}}{d t}=\beta_{\lambda_{\Sigma H}}= & \frac{1}{16 \pi^{2}}\left[4 \lambda_{\Sigma H}^{2}+6\left(\lambda_{H}+\lambda_{\Sigma}\right) \lambda_{\Sigma H}-\frac{\lambda_{\Sigma H}}{2}\left(3\left(g^{\prime}\right)^{2}+9 g^{2}+9 g_{X}^{2}+3\left(g_{X}^{\prime}\right)^{2}\right)\right. \\
\frac{d g_{X}}{d t}=\beta_{g_{X}}= & -\frac{1}{16 \pi^{2}} \frac{39}{6} g_{X}^{3}, \\
\frac{d g_{X}^{\prime}}{d t}=\beta_{g_{X}^{\prime}}= & \frac{1}{16 \pi^{2}} \frac{13}{6}\left(g_{X}^{\prime}\right)^{3}, \\
\frac{d Y_{\xi_{i}}}{d t}=\beta_{Y_{\xi_{i}}}= & \left.\left.\frac{1}{16 \pi^{2}} Y_{\xi_{i}}\left(\frac{3}{2}\left(Y_{\xi_{i}}^{2}-Y_{\chi_{i}}^{2}\right)+\sum_{j}\left(Y_{\xi_{j}}^{2}+Y_{\chi_{j}}^{2}\right)-\frac{9}{4} g_{X}^{2}-\frac{3}{4}\left(g_{X}^{\prime}\right)^{2}\right)\right)\right] \\
\frac{d Y_{\chi_{i}}}{d t}=\beta_{Y_{\chi_{i}}}= & \frac{1}{16 \pi^{2}} Y_{\chi_{i}}\left(\frac{3}{2}\left(Y_{\chi_{i}}^{2}-Y_{\xi_{i}}^{2}\right)+\sum_{j}\left(Y_{\xi_{j}}^{2}+Y_{\chi_{j}}^{2}\right)-\frac{9}{4} g_{X}^{2}-\frac{15}{4}\left(g_{X}^{\prime}\right)^{2}\right)
\end{aligned}
$$

\section{Loop function}

The loop function that enters the partial width of $h \rightarrow \gamma^{\prime} \gamma^{\prime}$ given in section 5 reads

$$
f(x)= \begin{cases}\arcsin ^{2} \sqrt{x} & \text { for } x \leq 1 \\ -\frac{1}{4}\left(\log \left(\frac{\sqrt{x}+\sqrt{x-1}}{\sqrt{x}-\sqrt{x-1}}\right)-i \pi\right)^{2} & \text { for } x>1\end{cases}
$$




\section{Dark matter annihilation}

In this appendix we give the annihilation cross section of the lightest neutral dark fermion $\xi_{1}$ into the charged dark fermions $\chi_{1}, \chi_{2}$, that are assumed to be lighter than $\xi_{1}$. Unsuppressed contributions come from s-channel exchange of a $Z^{\prime}$ boson and t-channel exchange of a $W^{\prime}$ boson. We find

$$
\begin{aligned}
(\sigma v)_{\xi_{1}} \simeq & \frac{1}{2 \pi} \frac{m_{\xi_{1}}^{2}}{w^{4}} \sqrt{1-\frac{m_{\chi_{1}}^{2}}{m_{\xi_{1}}^{2}}}\left(1+\frac{m_{\xi_{1}}^{2}}{m_{W^{\prime}}^{2}}-\frac{m_{\chi_{1}}^{2}}{m_{W^{\prime}}^{2}}\right)^{-2} \\
+ & \frac{1}{8 \pi} \frac{m_{\xi_{1}}^{2}}{w^{4}} \sum_{i=1,2} \sqrt{1-\frac{m_{\chi_{i}}^{2}}{m_{\xi_{1}}^{2}}}\left(1-4 s_{X}^{2}+8 s_{X}^{4}+\frac{m_{\chi_{i}}^{2}}{m_{\xi_{1}}^{2}} 2 s_{X}^{2}\left(2 s_{X}^{2}-1\right)\right) \\
& \times\left(\left(1-\frac{4 m_{\xi_{1}}^{2}}{m_{Z^{\prime}}^{2}}\right)^{2}+\frac{\Gamma_{Z^{\prime}}^{2}}{m_{Z^{\prime}}^{2}}\right)^{-1} \\
+ & \frac{1}{4 \pi} \frac{m_{\xi_{1}}^{2}}{w^{4}} \sqrt{1-\frac{m_{\chi_{1}}^{2}}{m_{\xi_{1}}^{2}}}\left(1-\frac{4 m_{\xi_{1}}^{2}}{m_{Z^{\prime}}^{2}}\right)\left(1-2 s_{X}^{2}+\frac{m_{\chi_{1}}^{2}}{m_{\xi_{1}}^{2}} s_{X}^{2}\right)^{-1} \\
& \times\left(1+\frac{m_{\xi_{1}}^{2}}{m_{W^{\prime}}^{2}}-\frac{m_{\chi_{1}}^{2}}{m_{W^{\prime}}^{2}}\right)^{-1}\left(\left(1-\frac{4 m_{\xi_{1}}^{2}}{m_{Z^{\prime}}^{2}}\right)^{2}+\frac{\Gamma_{Z^{\prime}}^{2}}{m_{Z^{\prime}}^{2}}\right)^{-1}
\end{aligned}
$$

The 1st line is the $W^{\prime}$ contribution, the 2nd and 3rd lines the $Z^{\prime}$ contribution and the 4 th and 5 th line the interference term.

The width of the $Z^{\prime}$ boson that enters the above expressions is given by

$$
\begin{gathered}
\Gamma_{Z^{\prime}} \simeq \sum_{i} \frac{g_{X}^{2}}{96 \pi c_{X}^{2}} m_{Z^{\prime}} \sqrt{1-\frac{4 m_{\xi_{i}}^{2}}{m_{Z^{\prime}}^{2}}}\left(1-4 s_{X}^{2}+8 s_{X}^{4}-\frac{m_{\xi_{i}}^{2}}{m_{Z^{\prime}}^{2}}\left(1+8 s_{X}^{2}-16 s_{X}^{4}\right)\right) \\
+\sum_{k} \frac{g_{X}^{2}}{96 \pi c_{X}^{2}} m_{Z^{\prime}} \sqrt{1-\frac{4 m_{\chi_{k}}^{2}}{m_{Z^{\prime}}^{2}}}\left(1-\frac{m_{\chi_{k}}^{2}}{m_{Z^{\prime}}^{2}}\right),
\end{gathered}
$$

where the sums over $i$ and $k$ run over those fermions with mass smaller than half of the $Z^{\prime}$ mass.

Open Access. This article is distributed under the terms of the Creative Commons Attribution License (CC-BY 4.0), which permits any use, distribution and reproduction in any medium, provided the original author(s) and source are credited.

\section{References}

[1] W.A. Bardeen, On naturalness in the standard model, FERMILAB-CONF-95-391.

[2] S.R. Coleman and E.J. Weinberg, Radiative Corrections as the Origin of Spontaneous Symmetry Breaking, Phys. Rev. D 7 (1973) 1888 [InSPIRE].

[3] E. Gildener and S. Weinberg, Symmetry Breaking and Scalar Bosons, Phys. Rev. D 13 (1976) 3333 [INSPIRE].

[4] R. Dermisek, T.H. Jung and H.D. Kim, Coleman-Weinberg Higgs, Phys. Rev. Lett. 113 (2014) 051801 [arXiv: 1308.0891] [INSPIRE]. 
[5] C.T. Hill, Is the Higgs Boson Associated with Coleman-Weinberg Dynamical Symmetry Breaking?, Phys. Rev. D 89 (2014) 073003 [arXiv:1401.4185] [INSPIRE].

[6] R. Hempfling, The Next-to-minimal Coleman-Weinberg model, Phys. Lett. B 379 (1996) 153 [hep-ph/9604278] [INSPIRE].

[7] R. Foot, A. Kobakhidze, K.L. McDonald and R.R. Volkas, A solution to the hierarchy problem from an almost decoupled hidden sector within a classically scale invariant theory, Phys. Rev. D 77 (2008) 035006 [arXiv:0709.2750] [INSPIRE].

[8] J.R. Espinosa, T. Konstandin, J.M. No and M. Quirós, Some Cosmological Implications of Hidden Sectors, Phys. Rev. D 78 (2008) 123528 [arXiv:0809.3215] [inSPIRE].

[9] J.R. Espinosa and M. Quirós, Novel Effects in Electroweak Breaking from a Hidden Sector, Phys. Rev. D 76 (2007) 076004 [hep-ph/0701145] [INSPIRE].

[10] S. Iso, N. Okada and Y. Orikasa, Classically conformal $B^{-}$L extended Standard Model, Phys. Lett. B 676 (2009) 81 [arXiv:0902.4050] [INSPIRE].

[11] R. Foot, A. Kobakhidze and R.R. Volkas, Stable mass hierarchies and dark matter from hidden sectors in the scale-invariant standard model, Phys. Rev. D 82 (2010) 035005 [arXiv: 1006.0131] [INSPIRE].

[12] L. Alexander-Nunneley and A. Pilaftsis, The Minimal Scale Invariant Extension of the Standard Model, JHEP 09 (2010) 021 [arXiv: 1006.5916] [INSPIRE].

[13] S. Iso and Y. Orikasa, TeV-scale B - L model with a flat Higgs potential at the Planck scale: in view of the hierarchy problem, PTEP 2013 (2013) 023B08 [arXiv:1210.2848] [INSPIRE].

[14] C. Englert, J. Jaeckel, V.V. Khoze and M. Spannowsky, Emergence of the Electroweak Scale through the Higgs Portal, JHEP 04 (2013) 060 [arXiv: 1301.4224] [INSPIRE].

[15] M. Farina, D. Pappadopulo and A. Strumia, A modified naturalness principle and its experimental tests, JHEP 08 (2013) 022 [arXiv: 1303.7244] [INSPIRE].

[16] M. Heikinheimo, A. Racioppi, M. Raidal, C. Spethmann and K. Tuominen, Physical Naturalness and Dynamical Breaking of Classical Scale Invariance, Mod. Phys. Lett. A 29 (2014) 1450077 [arXiv:1304.7006] [INSPIRE].

[17] T. Hambye and A. Strumia, Dynamical generation of the weak and Dark Matter scale, Phys. Rev. D 88 (2013) 055022 [arXiv: 1306.2329] [INSPIRE].

[18] C.D. Carone and R. Ramos, Classical scale-invariance, the electroweak scale and vector dark matter, Phys. Rev. D 88 (2013) 055020 [arXiv:1307.8428] [INSPIRE].

[19] A. Farzinnia, H.--J. He and J. Ren, Natural Electroweak Symmetry Breaking from Scale Invariant Higgs Mechanism, Phys. Lett. B 727 (2013) 141 [arXiv:1308.0295] [INSPIRE].

[20] V.V. Khoze, Inflation and Dark Matter in the Higgs Portal of Classically Scale Invariant Standard Model, JHEP 11 (2013) 215 [arXiv: 1308.6338] [INSPIRE].

[21] C. Tamarit, Running couplings with a vanishing scale anomaly, JHEP 12 (2013) 098 [arXiv: 1309.0913] [INSPIRE].

[22] E. Gabrielli, M. Heikinheimo, K. Kannike, A. Racioppi, M. Raidal and C. Spethmann, Towards Completing the Standard Model: Vacuum Stability, EWSB and Dark Matter, Phys. Rev. D 89 (2014) 015017 [arXiv: 1309.6632] [INSPIRE].

[23] T.G. Steele, Z.-W. Wang, D. Contreras and R.B. Mann, Viable dark matter via radiative symmetry breaking in a scalar singlet Higgs portal extension of the standard model, Phys. Rev. Lett. 112 (2014) 171602 [arXiv: 1310.1960] [INSPIRE]. 
[24] M. Hashimoto, S. Iso and Y. Orikasa, Radiative symmetry breaking at the Fermi scale and flat potential at the Planck scale, Phys. Rev. D 89 (2014) 016019 [arXiv:1310.4304] [InSPIRE].

[25] M. Holthausen, J. Kubo, K.S. Lim and M. Lindner, Electroweak and Conformal Symmetry Breaking by a Strongly Coupled Hidden Sector, JHEP 12 (2013) 076 [arXiv: 1310.4423] [INSPIRE].

[26] M. Hashimoto, S. Iso and Y. Orikasa, Radiative Symmetry Breaking from Flat Potential in various U(1)' models, Phys. Rev. D 89 (2014) 056010 [arXiv:1401.5944] [INSPIRE].

[27] S. Benic and B. Radovcic, Electroweak breaking and Dark Matter from the common scale, Phys. Lett. B 732 (2014) 91 [arXiv:1401.8183] [INSPIRE].

[28] V.V. Khoze, C. McCabe and G. Ro, Higgs vacuum stability from the dark matter portal, JHEP 08 (2014) 026 [arXiv: 1403.4953] [InSPIRE].

[29] A. Farzinnia and J. Ren, Higgs Partner Searches and Dark Matter Phenomenology in a Classically Scale Invariant Higgs Boson Sector, Phys. Rev. D 90 (2014) 015019 [arXiv: 1405.0498] [INSPIRE].

[30] G.M. Pelaggi, Predictions of a model of weak scale from dynamical breaking of scale invariance, arXiv:1406.4104 [INSPIRE].

[31] T. Hur, D.-W. Jung, P. Ko and J.Y. Lee, Electroweak symmetry breaking and cold dark matter from strongly interacting hidden sector, Phys. Lett. B 696 (2011) 262 [arXiv: 0709.1218] [INSPIRE].

[32] M. Lindner, S. Schmidt and J. Smirnov, Neutrino Masses and Conformal Electro-Weak Symmetry Breaking, JHEP 10 (2014) 177 [arXiv: 1405.6204] [INSPIRE].

[33] E.J. Chun, S. Jung and H.M. Lee, Radiative generation of the Higgs potential, Phys. Lett. B 725 (2013) 158 [arXiv:1304.5815] [INSPIRE].

[34] H. Davoudiasl and I.M. Lewis, Right-Handed Neutrinos as the Origin of the Electroweak Scale, Phys. Rev. D 90 (2014) 033003 [arXiv:1404.6260] [inSPIRE].

[35] K. Ishiwata, Dark Matter in Classically Scale-Invariant Two Singlets Standard Model, Phys. Lett. B 710 (2012) 134 [arXiv:1112.2696] [INSPIRE].

[36] J. Guo and Z. Kang, Higgs Naturalness and Dark Matter Stability by Scale Invariance, arXiv: 1401.5609 [INSPIRE].

[37] M.E. Machacek and M.T. Vaughn, Two Loop Renormalization Group Equations in a General Quantum Field Theory. 1. Wave Function Renormalization, Nucl. Phys. B 222 (1983) 83 [INSPIRE].

[38] M.E. Machacek and M.T. Vaughn, Two Loop Renormalization Group Equations in a General Quantum Field Theory. 2. Yukawa Couplings, Nucl. Phys. B 236 (1984) 221 [INSPIRE].

[39] M.E. Machacek and M.T. Vaughn, Two Loop Renormalization Group Equations in a General Quantum Field Theory. 3. Scalar Quartic Couplings, Nucl. Phys. B 249 (1985) 70 [INSPIRE].

[40] M.-x. Luo and Y. Xiao, Two loop renormalization group equations in the standard model, Phys. Rev. Lett. 90 (2003) 011601 [hep-ph/0207271] [INSPIRE].

[41] M.-x. Luo, H.-w. Wang and Y. Xiao, Two loop renormalization group equations in general gauge field theories, Phys. Rev. D 67 (2003) 065019 [hep-ph/0211440] [INSPIRE].

[42] M.-x. Luo and Y. Xiao, Renormalization group equations in gauge theories with multiple U(1) groups, Phys. Lett. B 555 (2003) 279 [hep-ph/0212152] [INSPIRE]. 
[43] S.P. Martin, Two loop effective potential for a general renormalizable theory and softly broken supersymmetry, Phys. Rev. D 65 (2002) 116003 [hep-ph/0111209] [INSPIRE].

[44] G. Degrassi et al., Higgs mass and vacuum stability in the Standard Model at NNLO, JHEP 08 (2012) 098 [arXiv: 1205.6497] [INSPIRE].

[45] D. Buttazzo et al., Investigating the near-criticality of the Higgs boson, JHEP 12 (2013) 089 [arXiv: 1307.3536] [INSPIRE].

[46] S. Weinberg and E. Witten, Limits on Massless Particles, Phys. Lett. B 96 (1980) 59 [INSPIRE].

[47] G. Dvali and C. Gomez, Black Hole Macro-Quantumness, arXiv:1212.0765 [InSPIRE].

[48] S. Dubovsky, V. Gorbenko and M. Mirbabayi, Natural Tuning: Towards A Proof of Concept, JHEP 09 (2013) 045 [arXiv: 1305.6939] [INSPIRE].

[49] G. Marques Tavares, M. Schmaltz and W. Skiba, Higgs mass naturalness and scale invariance in the UV, Phys. Rev. D 89 (2014) 015009 [arXiv:1308.0025] [INSPIRE].

[50] T. Hambye and M.H.G. Tytgat, Electroweak symmetry breaking induced by dark matter, Phys. Lett. B 659 (2008) 651 [arXiv:0707.0633] [INSPIRE].

[51] T.E. Clark, B. Liu, S.T. Love and T. ter Veldhuis, The Standard Model Higgs Boson-Inflaton and Dark Matter, Phys. Rev. D 80 (2009) 075019 [arXiv:0906.5595] [INSPIRE].

[52] R.N. Lerner and J. McDonald, Gauge singlet scalar as inflaton and thermal relic dark matter, Phys. Rev. D 80 (2009) 123507 [arXiv:0909.0520] [INSPIRE].

[53] M. Gonderinger, Y. Li, H. Patel and M.J. Ramsey-Musolf, Vacuum Stability, Perturbativity and Scalar Singlet Dark Matter, JHEP 01 (2010) 053 [arXiv:0910.3167] [INSPIRE].

[54] J. Elias-Miro, J.R. Espinosa, G.F. Giudice, H.M. Lee and A. Strumia, Stabilization of the Electroweak Vacuum by a Scalar Threshold Effect, JHEP 06 (2012) 031 [arXiv: 1203.0237] [INSPIRE].

[55] O. Lebedev, On Stability of the Electroweak Vacuum and the Higgs Portal, Eur. Phys. J. C 72 (2012) 2058 [arXiv:1203.0156] [INSPIRE].

[56] B. Batell, S. Jung and H.M. Lee, Singlet Assisted Vacuum Stability and the Higgs to Diphoton Rate, JHEP 01 (2013) 135 [arXiv:1211.2449] [INSPIRE].

[57] J. Kubo, K.S. Lim and M. Lindner, Electroweak Symmetry Breaking via QCD, Phys. Rev. Lett. 113 (2014) 091604 [arXiv: 1403.4262] [INSPIRE].

[58] M. Sher, Electroweak Higgs Potentials and Vacuum Stability, Phys. Rept. 179 (1989) 273 [INSPIRE].

[59] ATLAS collaboration, Updated coupling measurements of the Higgs boson with the ATLAS detector using up to 25 $\mathrm{fb}^{-1}$ of proton-proton collision data, ATLAS-CONF-2014-009 (2014).

[60] CMS collaboration, Precise determination of the mass of the Higgs boson and studies of the compatibility of its couplings with the standard model, CMS-PAS-HIG-14-009.

[61] ATLAS collaboration, Search for Invisible Decays of a Higgs Boson Produced in Association with a Z Boson in ATLAS, Phys. Rev. Lett. 112 (2014) 201802 [arXiv:1402.3244] [INSPIRE].

[62] CMS collaboration, Search for invisible decays of Higgs bosons in the vector boson fusion and associated ZH production modes, Eur. Phys. J. C 74 (2014) 2980 [arXiv:1404.1344] [INSPIRE].

[63] CMS collaboration, Search for an Invisible Higgs Boson, CMS-PAS-HIG-13-013. 
[64] N. Zhou, Z. Khechadoorian, D. Whiteson and T.M.P. Tait, Bounds on Invisible Higgs boson Decays from $t \bar{t} H$ Production, arXiv:1408.0011 [INSPIRE].

[65] CMS collaboration, Search for top-squark pair production in the single-lepton final state in pp collisions at $\sqrt{s}=8 \mathrm{TeV}$, Eur. Phys. J. C 73 (2013) 2677 [arXiv:1308.1586] [InSPIRE].

[66] S. Dawson et al., Working Group Report: Higgs Boson, arXiv:1310.8361 [INSPIRE].

[67] CMS collaboration, Measurement of Higgs boson production and properties in the $W W$ decay channel with leptonic final states, JHEP 01 (2014) 096 [arXiv:1312.1129] [INSPIRE].

[68] CMS collaboration, Measurement of the properties of a Higgs boson in the four-lepton final state, Phys. Rev. D 89 (2014) 092007 [arXiv: 1312.5353] [INSPIRE].

[69] ATLAS collaboration, Measurements of the properties of the Higgs-like boson in the $W W^{(*)} \rightarrow \ell \nu \ell \nu$ decay channel with the ATLAS detector using $25 \mathrm{fb}^{-1}$ of proton-proton collision data, ATLAS-CONF-2013-030 (2013).

[70] ATLAS collaboration, Measurements of the properties of the Higgs-like boson in the four lepton decay channel with the ATLAS detector using $25 \mathrm{fb}-1$ of proton-proton collision data, ATLAS-CONF-2013-013 (2013).

[71] J. Fan, A. Katz, L. Randall and M. Reece, Double-Disk Dark Matter, Phys. Dark Univ. 2 (2013) 139 [arXiv: 1303.1521] [INSPIRE].

[72] L. Ackerman, M.R. Buckley, S.M. Carroll and M. Kamionkowski, Dark Matter and Dark Radiation, Phys. Rev. D 79 (2009) 023519 [arXiv:0810.5126] [INSPIRE].

[73] J.L. Feng, M. Kaplinghat, H. Tu and H.-B. Yu, Hidden Charged Dark Matter, JCAP 07 (2009) 004 [arXiv: 0905.3039] [INSPIRE].

[74] A.H.G. Peter, M. Rocha, J.S. Bullock and M. Kaplinghat, Cosmological Simulations with Self-Interacting Dark Matter II: Halo Shapes vs. Observations, Mon. Not. Roy. Astron. Soc. 430 (2013) 105 [arXiv:1208.3026] [INSPIRE].

[75] M. Markevitch et al., Direct constraints on the dark matter self-interaction cross-section from the merging galaxy cluster 1E0657-56, Astrophys. J. 606 (2004) 819 [astro-ph/0309303] [INSPIRE].

[76] S.W. Randall, M. Markevitch, D. Clowe, A.H. Gonzalez and M. Bradac, Constraints on the Self-Interaction Cross-Section of Dark Matter from Numerical Simulations of the Merging Galaxy Cluster 1E 0657-56, Astrophys. J. 679 (2008) 1173 [arXiv:0704.0261] [INSPIRE].

[77] F.-Y. Cyr-Racine, R. de Putter, A. Raccanelli and K. Sigurdson, Constraints on Large-Scale Dark Acoustic Oscillations from Cosmology, Phys. Rev. D 89 (2014) 063517 [arXiv: 1310.3278] [INSPIRE].

[78] J. Giedt, A.W. Thomas and R.D. Young, Dark matter, the CMSSM and lattice QCD, Phys. Rev. Lett. 103 (2009) 201802 [arXiv:0907.4177] [INSPIRE].

[79] A. Crivellin, M. Hoferichter and M. Procura, Accurate evaluation of hadronic uncertainties in spin-independent WIMP-nucleon scattering: Disentangling two- and three-flavor effects, Phys. Rev. D 89 (2014) 054021 [arXiv: 1312.4951] [InSPIRE].

[80] XENON100 collaboration, E. Aprile et al., Dark Matter Results from 225 Live Days of XENON100 Data, Phys. Rev. Lett. 109 (2012) 181301 [arXiv:1207.5988] [InSPIRE].

[81] LUX collaboration, D.S. Akerib et al., First results from the LUX dark matter experiment at the Sanford Underground Research Facility, Phys. Rev. Lett. 112 (2014) 091303 [arXiv: 1310.8214] [INSPIRE]. 
[82] XENON1T collaboration, E. Aprile, The XENON1T Dark Matter Search Experiment, Springer Proc. Phys. C12-02-22 (2013) 93 [arXiv:1206.6288] [INSPIRE].

[83] D.C. Malling et al., After LUX: The LZ Program, arXiv:1110.0103 [inSPIRE].

[84] R.H. Cyburt, B.D. Fields, K.A. Olive and E. Skillman, New BBN limits on physics beyond the standard model from He-4, Astropart. Phys. 23 (2005) 313 [astro-ph/0408033] [INSPIRE].

[85] Planck collaboration, P.A.R. Ade et al., Planck 2013 results. XVI. Cosmological parameters, Astron. Astrophys. 571 (2014) A16 [arXiv:1303.5076] [InSPIRE].

[86] U. França, R.A. Lineros, J. Palacio and S. Pastor, Probing interactions within the dark matter sector via extra radiation contributions, Phys. Rev. D 87 (2013) 123521 [arXiv: 1303.1776] [INSPIRE].

[87] G. Mangano, G. Miele, S. Pastor, T. Pinto, O. Pisanti P.D. Serpico, Relic neutrino decoupling including flavor oscillations, Nucl. Phys. B 729 (2005) 221 [hep-ph/0506164] [INSPIRE].

[88] J.L. Feng et al., Planning the Future of U.S. Particle Physics (Snowmass 2013): Chapter 4: Cosmic Frontier, arXiv:1401.6085 [INSPIRE].

[89] B. Famaey, Gaia and the dynamics of the Galaxy, SF2A-2012: Proceedings of the Annual meeting of the French Society of Astronomy and Astrophysics, [arXiv:1209.5753] [INSPIRE].

[90] M. Trodden, Electroweak baryogenesis, Rev. Mod. Phys. 71 (1999) 1463 [hep-ph/9803479] [INSPIRE].

[91] D.E. Morrissey and M.J. Ramsey-Musolf, Electroweak baryogenesis, New J. Phys. 14 (2012) 125003 [arXiv: 1206.2942] [inSPIRE].

[92] J.R. Espinosa, T. Konstandin and F. Riva, Strong Electroweak Phase Transitions in the Standard Model with a Singlet, Nucl. Phys. B 854 (2012) 592 [arXiv:1107.5441] [INSPIRE].

[93] S. Profumo, M.J. Ramsey-Musolf and G. Shaughnessy, Singlet Higgs phenomenology and the electroweak phase transition, JHEP 08 (2007) 010 [arXiv:0705.2425] [INSPIRE].

[94] H.H. Patel and M.J. Ramsey-Musolf, Stepping Into Electroweak Symmetry Breaking: Phase Transitions and Higgs Phenomenology, Phys. Rev. D 88 (2013) 035013 [arXiv:1212.5652] [INSPIRE].

[95] E.J. Weinberg and A.-q. Wu, Understanding complex perturbative effective potentials, Phys. Rev. D 36 (1987) 2474 [INSPIRE].

[96] S.P. Martin, Taming the Goldstone contributions to the effective potential, Phys. Rev. D 90 (2014) 016013 [arXiv:1406.2355] [INSPIRE].

[97] J. Elias-Miro, J.R. Espinosa and T. Konstandin, Taming Infrared Divergences in the Effective Potential, JHEP 08 (2014) 034 [arXiv:1406.2652] [INSPIRE].

[98] A. Andreassen, W. Frost and M.D. Schwartz, Consistent Use of the Standard Model Effective Potential, arXiv:1408.0292 [INSPIRE]. 